\title{
A novel approach to MADM problems using Fermatean fuzzy Hamacher prioritized aggre- gation operators
}

\author{
${ }^{a}$ Asmatullah Jan, ${ }^{a}$ Asghar Khan ${ }^{1},{ }^{b}$ Waqar Khan and ${ }^{a}$ Minhaj Afridi \\ ${ }^{a}$ Department of Mathematics, Abdul Wali Khan University, Mardan, KP, Pakistan \\ ${ }^{b}$ School of Mathematics and Statistics, Yancheng Teachers University, Yancheng, China
}

\begin{abstract}
A generalized form of union and intersection on FFS can be formulated from a generalized t-norm (TN) and t-conorm (TCN). Hamacher operations such as Hamacher product and Hamacher sum, are good alternatives to produce such product and sum. The Hamacher operations can generate more flexible and more accurate results in decision making process due to the working parameter involved in these operations. The intuitionistic fuzzy set, brifely as; IFS and its extension involving Pythagorean fuzzy set (PFS) and Fermatean fuzzy set (FFS), are all effective tools to express uncertain and incomplete cognitive information with membership, nonmembership and hesitancy degrees. The Fermatean fuzzy set (FF-set) carries out uncertain and imprecise information smartly in exercising decision-making than IFS and PFS. By adjusting the prioritization of attributes in FF-environment, in this course of this article, we first device new operations on $\mathrm{FF}$ information using prioritized attributes and by employing HTN and HTCN, we discuss the basic operations. Induced by the Hamacher operations and FF-set, we propose FF Hamacher arithmetic and also geometric aggregation operators (AOs). In the first section, we introduce the concepts of an FF Hamacher prioritized $\mathrm{AO}$, and FF Hamacher prioritized weighted AO. In the second part, we develop FF Hamacher prioritized geometric operator (GO), and FF Hamacher prioritized weighted GO. We study essential properties and a few special cases of our newly proposed operators. Then, we make use of these proposed operators in developing tools which are key factors in solving the FF multi-attribute decision-making situations with prioritization. The university selection phenomena is considered as a direct application for analysis and to demonstrate the practicality and efficacy of our proposed model. The working parameter considered in these AOs is analyzed in different existing and proposed AOs. Further, comparison analysis is conducted for the authenticity of proposed \& existing operators.
\end{abstract}

\footnotetext{
${ }^{1}$ Corresponding author's e-mail: asghar@awkum.edu.pk (A. Khan), minhajafridi342@gmail.com (M. Afridi) waqarkhan@gmail.com (W. Khan), ajanmath24@gmail.com (A. Jan)
} 
Keywords: MADM; FF Hamacher prioritized average (FFHPA) operator; FF Hamacher prioritized weighted average (FFHPWA) operator; FF Hamacher prioritized geometric (FFHPG) operator; FF Hamacher prioritized weighted geometric (FFHPWG) operator.

\section{Introduction}

Pythagorean fuzzy sets (PFSs) [21,22], an augmentation of intuitionistic fuzzy sets (IFSs), tremendously have attracted many potential researchers in recent times. Yager [23] was the first researcher who built a helpful decision technique which based on the Pythagorean fuzzy information operators to administer with MCDM situatoins involving Pythagorean fuzzy information. In [27], Yager and Abbasov treated the Pythagorean membership grades (PMGs) and they found it identical with PFSs. Their work also showed the relation between the PMGs and the complex numbers. Reformat and Yager [23] exerted PFNs to build a system for dealing with collaborative based recommender. Gou et al. [5] studied Pythagorean fuzzy mappings and investigated fundamental properties called derivability, continuity, and differentiability. Zeng et al. [29] introduced an aggregation procedure involving PFS and applied its notion in solving MADM. Zhang [30] proposed an approach to MCDM problems in terms of the idea of similarity measure for Pythagorean fuzzy sets. PFSs have been successfully introduced in different research area, in particular, Garg used PFSs in investment decision process (see Garg, [4]; Peng and Yang, [11]), utilized PFSs in the candidate selection procedure for Asian Infrastructure Investment Bank (Ren et al., [13]) and the service excellence of national airlines (Zhang and $\mathrm{Xu},[31])$. Senapati and Yager $[14,15]$ discussed a numerical case to validate the rationality of the concept of FFS: when one desires to show his inclination for the degree of an alternative $x_{i}$ under certain criterion $C_{j}$, e.g., one may allow the degree to the extent in a way that the alternative $x_{i}$ satisfies the criterion $C_{j}$ as 0.8 , and similarly when the alternative $x_{i}$ dissatisfies the criterion $C_{j}$ as 0.7 . We can precisely get $0.8+0.7>1$, and consequently, the boundary condition of an IFS is not satisfied. Also, for the case when $(0.8)^{2}+(0.7)^{2}=0.64+0.49=1.13>1$, which does not carry out the boundary condition of a PFS. However, it clear to see $(0.8)^{3}+(0.7)^{3}=0.512+0.343=0.855<1$, which is an appropriate reason for the introduction of a new division of fuzzy set, called Fermatean fuzzy set. It is also important to mention that the class of this type of fuzzy set has more ability to capture the uncertainties as compared to IFSs and PFSs, and are qualified to handle higher degree of vagueness. MADM has been extensively used in many area of sciences, for example, (Xu \& Xia; Xu \& Chen; Xu [24-26]) introduced the intuitionistic fuzzy weighted averaging (IFWA) operator, intuitionistic fuzzy ordered weighted averaging (IFOWA) operator and the intuitionistic fuzzy hybrid aggregation (IFHA) operator.

The fuzzy information aggregation operators are necessarily appealing and significant research topics and are given profound regard among the researchers. Various forms of generalizations of T-norms and T-conorms exist in text, such as Archimedean T-norms and Tconorms, Hamacher T-norms and T-conorms, Algebraic T-norms and T-conorms, Einstein T- 
norms and T-conorms, Frank T-norms and T-conorms and Dombi T-norms and T-conorms. Liu [8] used Hamacher aggregation operators in interval valued intuitionistic fuzzy numbers (IVIFNs) and discussed MAGDM techniques. Zhao and Wei [32] initiated Einstein hybrid aggregation operators for IFNs and applied it to multi-attribute decision-making method. Wei et al. [33] studied multi-attribute decision-making problems by proposing the bipolar fuzzy Hamacher arithmetic and geometric aggregation operators and discussed the basic properties of these proposed operators. Hamacher T-conorm and $\mathrm{T}$-norm, which are the generalization of algebraic and Einstein T-conorm and T-norm [3], are more universal and adaptable. The importance of aggregation operators research based on Hamacher operations and their application to MADM problems is considerable significant. Xiao [20] gave induced intervalvalued intuitionistic fuzzy Hamacher ordered weighted geometric (IIVIFHOWG) operator. $\mathrm{Li}[7]$ studied interval-valued intuitionistic fuzzy sets with various operations, known as the Hamacher sum and the Hamacher product, and introduced the interval-valued intuitionistic fuzzy Hamacher correlated averaging (IVIFHCA) operator. Tan et al. [17] developed hesitant fuzzy Hamacher aggregation operators for multi-attribute decision-making.

Senapati and Yager [34] introduced four new types of weighted aggregation operators for FFS, namely, Fermatean fuzzy weighted average (FFWA) operator, Fermatean fuzzy weighted geometric (FFWG) operator, Fermatean fuzzy weighted power average (FFWPA) operator, and Fermatean fuzzy weighted power geometric (FFWPG) operator. Recently, in a paper [42], of Aydemir \& Gunduz discussed TOPSIS method in terms of Dombi aggregation operators based on FF-sets and gave a complete overview of FF-sets in the frame-work of Dombi operations. In [43], the authors have extended FF-set into Hamacher operations and investigated the basic properties of FF-sets in Hamacher operations. Some practical examples of real world scenario were discussed as practical example for the validation of the theory. Keeping in view the work on FF-set, we intend to extend the work of [43] and propose a set of new aggregation operators in Fermatean fuzzy environment based upon the Hamacher operations with the prioritization of attributes. The organization and novel contributions of this article are mentioned as:

$\triangleright$ The FF-set has more potential than the traditional IFS and PFS for decision makers to study the uncertain situation in the real world problems.

$\triangleright$ The flexibility parameter involved in Hamacher operations has the ability to produce more accurate results in a decision process.

$\triangleright$ The proposed model can be applied in situations where the traditional models of IFS and PFS are failed.

$\triangleright$ The prioritization factor of attributes make the proposed operators more advanced in modern decision process.

The remaining parts of the paper are organized in the following lines.

The second section, in brief, recalls basic knowledge of the IFS, PFSs, and FFSs and the elemntary operational laws of FFSs. In Sect. 3, we develop Fermatean fuzzy Hamacher prioritized average (FFHPA) operator, and Fermatean fuzzy Hamacher prioritized weighted average 
(FFHPWA) operator, Fermatean fuzzy Hamacher prioritized geometric (FFHPG) operator, and Fermatean fuzzy Hamacher prioritized weighted geometric (FFHPWG) operator. In Sect. 4, we make a use of these operators to develop tools that are handy in solving the Fermatean fuzzy multi-attribute decision-making problems. A case study example of the university selection committee is analyzed in Sect. 5, and some comparison of proposed operators are studied. The comparison of proposed and existing operators is studied and some future directions are given at the end of the paper.

\section{Preliminaries and basic results}

In this section, we sum up requisite knowledge associated with IFS, PFS and FFS along with corresponding operations and related properties. We will consider more familiarized ideas, which are useful in the sequential analysis.

Definition 2.1. $[1,2]$ For a universe $X$, intuitionistic fuzzy set (IFS) $\widetilde{A}$ is an expression of the form

$$
\widetilde{A}=\left\{x, \widetilde{\alpha}_{\widetilde{A}}(x), \widetilde{\beta}_{\widetilde{A}}(x): x \in X\right\}
$$

where $\widetilde{\alpha}_{\widetilde{A}}(x) \in[0,1]$ is known as the "degree of membership of $\widetilde{A}$ ", and $\widetilde{\beta}_{\widetilde{A}}(x) \in[0,1]$ is called the "degree of non-membership of $\widetilde{A}$ ", and $\widetilde{\alpha}_{\widetilde{A}}(x), \widetilde{\beta}_{\widetilde{A}}(x)$ satisfy the following condition: $0 \leq \widetilde{\alpha}_{\widetilde{A}}(x)+\widetilde{\beta}_{\widetilde{A}}(x) \leq 1$, for all $x \in X$. Apparently, when $\widetilde{\beta}_{\widetilde{A}}(x)=1-\widetilde{\alpha}_{\widetilde{A}}(x)$, for all $x \in X, \widetilde{A}$ turns to be a fuzzy set.

Definition 2.2. [4] A Pythagorean fuzzy set (PFS) $P$ on the universal $X$ is an object of the form

$$
P=\left\{x, \alpha_{P}(x), \beta_{P}(x): x \in X\right\}
$$

where $\alpha_{P}: X \longrightarrow[0,1]$ is termed as the "degree of membership of $P$ ", and $\beta_{P}$ : $X \longrightarrow[0,1]$ is called the "degree of non-membership of $P$ ", and $\alpha_{P}(x), \beta_{P}(x)$ satisfies the condition: $0 \leq\left(\alpha_{P}(x)\right)^{2}+\left(\beta_{P}(x)\right)^{2} \leq 1$ for all $x \in X$. For PFS, $P$ and $x \in X$, $\pi(x)=\sqrt{1-\left(\alpha_{P}(x)\right)^{2}-\left(\beta_{P}(x)\right)^{2}}$ is the indeterminacy of $x$ to $P$.

Definition 2.3. [14] A Fermatean fuzzy set (FFS) defined on a nonempty set $X$ is a structure of the form given as

$$
\boldsymbol{F}=\left\{\left\langle x, \alpha_{\boldsymbol{F}}(x), \beta_{\boldsymbol{F}}(x)\right\rangle: x \in X\right\}
$$

where $\alpha_{\boldsymbol{F}}: X \longrightarrow[0,1]$, and $\beta_{\boldsymbol{F}}: X \longrightarrow[0,1]$, respectively are the degree of membership and non-membership of every element $x \in X$ for the set $\boldsymbol{F}$. The condition $0 \leq$ $\left(\alpha_{\boldsymbol{F}}(x)\right)^{3}+\left(\beta_{\boldsymbol{F}}(x)\right)^{3} \leq 1$, holds for all $x \in X$. For an FFS, $\boldsymbol{F}$ and $x \in X$, the function $\pi(x)=\sqrt{1-\left(\alpha_{\boldsymbol{F}}(x)\right)^{3}-\left(\beta_{\boldsymbol{F}}(x)\right)^{3}}$ is the indeterminacy of $x$ to $\boldsymbol{F}$.

For simplicity, we use $\boldsymbol{F}=(\mu, \nu)$ for an FFS $\left\{\left\langle\left\langle x, \alpha_{\boldsymbol{F}}(x), \beta_{\boldsymbol{F}}(x)\right\rangle: x \in X\right\rangle\right\}$ and call it a 
Fermatean fuzzy element (FFE) or Fermatean fuzzy number (FFN).

Hierarchy of fuzzy numbers

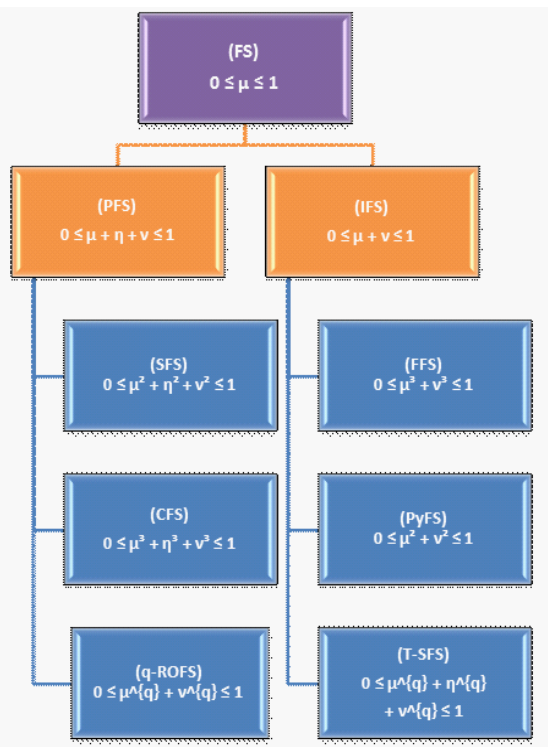

We shall notice that the Fermatean membership grades (FMGs) is greater than the Pythagorean membership grades (PMGs) and intuitionistic membership grades (IMGs), respectively.

Theorem 2.4. [14] The set of FMGs is larger than the set of PMGs and IMGs.

Definition 2.5. [14] Let $\boldsymbol{F}=(\mu, \nu), \boldsymbol{F}_{1}=\left(\mu_{1}, \nu_{1}\right)$, and $\boldsymbol{F}_{2}=\left(\mu_{2}, \nu_{2}\right)$, be any three FFNs, then their set operations are defined as in the following:

(i) $\boldsymbol{F}_{1} \cap \boldsymbol{F}_{2}=\left(\min \left\{\mu_{1}, \mu_{2}\right\}, \max \left\{\nu_{1}, \nu_{2}\right\}\right)$;

(ii) $\boldsymbol{F}_{1} \cup \boldsymbol{F}_{2}=\left(\max \left\{\mu_{1}, \mu_{2}\right\}, \max \left\{\nu_{1}, \nu_{2}\right\}\right)$;

(iii) $\boldsymbol{F}_{1} \subseteq \boldsymbol{F}_{2}$ if and only if $\mu_{1} \leq \mu_{2}$, and $\nu_{1} \geq \nu_{2}$;

(iv) $\boldsymbol{F}^{c}=(\nu, \mu)$.

Definition 2.6. [19]. Let $\boldsymbol{F}=(\mu, \nu), \boldsymbol{F}_{1}=\left(\mu_{1}, \nu_{1}\right)$, and $\boldsymbol{F}_{2}=\left(\mu_{2}, \nu_{2}\right)$, be any three FFEs, and $\xi>0$, then the following operations hold true.

(i) $\boldsymbol{F}_{1} \oplus \boldsymbol{F}_{2}=\left(\sqrt[3]{\mu_{1}^{3}+\mu_{2}^{3}-\mu_{1}^{3} \mu_{2}^{3}}, \nu_{1} \nu_{2}\right)$;

(ii) $\boldsymbol{F}_{1} \otimes \boldsymbol{F}_{2}=\left(\mu_{1} \mu_{2}, \sqrt[3]{\nu_{1}^{3}+\nu_{2}^{3}-\nu_{1}^{3} \nu_{2}^{3}}\right)$;

(iii) $\xi(\boldsymbol{F})=\left(\sqrt[3]{1-\left(1-\mu^{3}\right)^{\xi}}, \nu^{\xi}\right)$;

(iv) $(\boldsymbol{F})^{\xi}=\left(\mu^{\xi}, \sqrt[3]{1-\left(1-\nu^{3}\right)^{\xi}}\right)$. 
Theorem 2.7. [19]. For three FFEs $\boldsymbol{F}=(\mu, \nu), \boldsymbol{F}_{1}=\left(\mu_{1}, \nu_{1}\right)$, and $\boldsymbol{F}_{2}=\left(\mu_{2}, \nu_{2}\right)$, the following properties are valid:

(i) $\boldsymbol{F}_{1} \oplus \boldsymbol{F}_{2}=\boldsymbol{F}_{2} \oplus \boldsymbol{F}_{1}$;

(ii) $\boldsymbol{F}_{1} \otimes \boldsymbol{F}_{2}=\boldsymbol{F}_{2} \otimes \boldsymbol{F}_{1}$;

(iii) $\xi\left(\boldsymbol{F}_{1} \oplus \boldsymbol{F}_{2}\right)=\xi \boldsymbol{F}_{1} \oplus \xi \boldsymbol{F}_{2}, \xi>0$;

(iv) $\left(\xi_{1}+\xi_{2}\right) \boldsymbol{F}=\xi_{1} \boldsymbol{F} \oplus \xi_{2} \boldsymbol{F}, \xi_{1}, \xi_{2}>0$;

(v) $\left(\boldsymbol{F}_{1} \otimes \boldsymbol{F}_{2}\right)^{\xi}=\boldsymbol{F}_{1}^{\xi} \otimes \boldsymbol{F}_{2}^{\xi}, \xi>0$;

(vi) $\boldsymbol{F}^{\xi_{1}} \oplus \boldsymbol{F}^{\xi_{2}}=\boldsymbol{F}^{\xi_{1}+\xi_{2}}, \xi_{1}, \xi_{2}>0$.

In order to grade FFEs, the rating function (briefly as rt) for FFEs is defined as:

Definition 2.8. [19] For FFE, $\boldsymbol{F}=(\mu, \nu)$, the rating function of $\boldsymbol{F}$ can be defined as follows:

$$
\operatorname{rt}(\boldsymbol{F})=\frac{1+\mu^{3}-\nu^{3}}{2} \text {. }
$$

In particular $\operatorname{rt}(\boldsymbol{F})= \begin{cases}1, & \text { if } \boldsymbol{F}=(1,0) \\ -1, & \text { if } \boldsymbol{F}=(0,1)\end{cases}$

In the following, we examine a comparison relation for Fermatean fuzzy elements.

Definition 2.9. [19] Let $\boldsymbol{F}_{1}=\left(\mu_{1}, \nu_{1}\right)$, and $\boldsymbol{F}_{2}=\left(\mu_{2}, \nu_{2}\right)$, be any two FFEs and let $\operatorname{rt}\left(\boldsymbol{F}_{1}\right)$ and $\operatorname{rt}\left(\boldsymbol{F}_{2}\right)$ be the respective ratings of $\boldsymbol{F}_{1}$ and $\boldsymbol{F}_{2}$, then

(i) $\operatorname{rt}\left(\boldsymbol{F}_{1}\right)>\operatorname{rt}\left(\boldsymbol{F}_{2}\right)$, then $\boldsymbol{F}_{1}$ is superior than $\boldsymbol{F}_{2}$ and denoted by $\boldsymbol{F}_{1} \succ \boldsymbol{F}_{2}$;

(ii) $\operatorname{rt}\left(\boldsymbol{F}_{1}\right)=\operatorname{rt}\left(\boldsymbol{F}_{2}\right)$, then $\boldsymbol{F}_{1}$ and $\boldsymbol{F}_{2}$ are equivalent, denoted by $F_{1} \sim F_{2}$.

Definition 2.10. [19] Let $F=(\mu, \nu)$, be a FFE. The accuracy function of $F$ can be defined as follows:

$$
\operatorname{Acc}(\boldsymbol{F})=\mu^{3}+\nu^{3} \in[0,1] .
$$

Using the analogy of rating function (briefly as; rt) and accuracy function (briefly as; Acc), we give a complete criterion for the ranking of FFEs in the following.

Definition 2.11. [19] Let $\boldsymbol{F}_{1}=\left(\mu_{1}, \nu_{1}\right)$, and $\boldsymbol{F}_{2}=\left(\mu_{2}, \nu_{2}\right)$, be any two FFEs and let $\operatorname{rt}\left(\boldsymbol{F}_{i}\right)$ and $\operatorname{Acc}\left(\boldsymbol{F}_{i}\right)(i=1,2)$ be the respective ratings and accuracies of $\boldsymbol{F}_{1}$ and $\boldsymbol{F}_{2}$, then

(I) $\operatorname{rt}\left(\boldsymbol{F}_{1}\right)<\operatorname{rt}\left(\boldsymbol{F}_{2}\right) \Longrightarrow \boldsymbol{F}_{1} \prec \boldsymbol{F}_{2}$;

(II) $\operatorname{rt}\left(\boldsymbol{F}_{1}\right)>\operatorname{rt}\left(\boldsymbol{F}_{2}\right) \Longrightarrow \boldsymbol{F}_{1} \succ \boldsymbol{F}_{2}$;

(III) If $\operatorname{rt}\left(\boldsymbol{F}_{1}\right)=\operatorname{rt}\left(\boldsymbol{F}_{2}\right)$, then

(i) $\operatorname{Acc}\left(\boldsymbol{F}_{1}\right)<\operatorname{Acc}\left(\mathbf{F}_{2}\right) \Longrightarrow \boldsymbol{F}_{1}<\boldsymbol{F}_{2}$;

(ii) $\operatorname{Acc}\left(\boldsymbol{F}_{1}\right)>\operatorname{Acc}\left(\boldsymbol{F}_{2}\right) \Longrightarrow \boldsymbol{F}_{1}>\boldsymbol{F}_{2}$;

(iii) $\operatorname{Acc}\left(\boldsymbol{F}_{1}\right)=\operatorname{Acc}\left(\boldsymbol{F}_{2}\right) \Longrightarrow \boldsymbol{F}_{1} \sim \boldsymbol{F}_{2}$. 


\subsection{Hamacher operations}

Hamacher proposed generalized form of T-norm as well as T-conorm, called Hamacher operations, which consists of Hamacher product and Hamacher sum. These are the respective blueprints of the well known T-norm and $\mathrm{T}$-conorm, mentioned in the definition below.

Definition 2.12. [6] Assume $a_{1}, b_{1}, a_{2}, b_{2} \in R$. Then, Hamacher T-norms (HT-norms) and Hamacher T-conorms (HT-conorms) are expressed as:

$$
\begin{gathered}
T\left(a_{1}, b_{1}\right)=a_{1} \otimes b_{1}=\frac{a_{1} b_{1}}{\lambda+(1-\lambda)\left(a_{1}+b_{1}-a_{1} b_{1}\right)}, \lambda>0 \\
T^{*}\left(a_{2}, b_{2}\right)=a_{2} \oplus b_{2}=\frac{a_{2}+b_{2}-a_{2} b_{2}-(1-\lambda) a_{2} b_{2}}{1-(1-\lambda) a_{2} b_{2}}, \lambda>0
\end{gathered}
$$

Particularly, for $\lambda=1$, HT-norm and HT-conorm take the following forms:

$$
\begin{aligned}
T\left(a_{1}, b_{1}\right) & =a_{1} \otimes b_{1}=a_{1} b_{1} \\
T^{*}\left(a_{2}, b_{2}\right) & =a_{2} \oplus b_{2}=a_{2}+b_{2}-a_{2} b_{2}
\end{aligned}
$$

and these are algebraically equivalent. When $\lambda=2$,

$$
\begin{aligned}
T\left(a_{1}, b_{1}\right) & =a_{1} \otimes b_{1}=\frac{a_{1} b_{1}}{1+\left(1-a_{1}\right)\left(1-b_{1}\right)} \\
T^{*}\left(a_{2}, b_{2}\right) & =a_{2} \oplus b_{2}=\frac{a_{2}+b_{2}}{1+a_{2} b_{2}}
\end{aligned}
$$

Eqs. (5) and (6), respectively are known as the Einstein T-norm and Einstein T-conorm.

\section{FF Hamacher operators}

In this section, utilizing the notion of HTN and HTCN, we explain Hamacher operations with respect to FFEs. We propose the Hamacher arithmetic AOs with FFEs. In this regard, the operation rules for FF Hamacher operation are recalled in the following definition.

Definition 3.1. Let $\boldsymbol{F}_{j}=\left(\mu_{j}, \nu_{j}\right)(j=1,2)$ be a array of FFEs, $\lambda>0, \kappa>0$, then, the fundamental Hamacher operations for FFEs are introduced as:

(i) $\boldsymbol{F}_{1} \oplus \boldsymbol{F}_{2}=\left(\sqrt[3]{\frac{\mu_{1}^{3}+\left(\mu_{2}\right)^{3}-\left(\mu_{1}\right)^{3} \mu_{2}^{3}-(1-\lambda) \mu_{1}^{3} \mu_{2}^{3}}{1-(1-\lambda) \mu_{1}^{3} \mu_{2}^{3}}}, \frac{\nu_{1} \nu_{2}}{\sqrt[3]{\lambda+(1-\lambda)\left(\nu_{1}^{3}+\nu_{2}^{3}-\nu_{1}^{3} \nu_{2}^{3}\right)}}\right)$;

(ii) $\boldsymbol{F}_{1} \otimes \boldsymbol{F}_{2}=\left(\frac{\mu_{1} \mu_{2}}{\sqrt[3]{\lambda+(1-\lambda)\left(\left(\mu_{1}\right)^{3}+\left(\mu_{2}\right)^{3}-\left(\mu_{1}\right)^{3}\left(\mu_{2}\right)^{3}\right)}}, \sqrt[3]{\frac{\left(\nu_{1}\right)^{3}+\left(\nu_{2}\right)^{3}-\left(\nu_{1}\right)^{3}\left(\nu_{2}\right)^{3}-(1-\lambda)\left(\nu_{1}\right)^{3}\left(\nu_{2}\right)^{3}}{1-(1-\lambda)\left(\nu_{1}\right)^{3}\left(\nu_{2}\right)^{3}}}\right)$; 
(iii) $\kappa\left(\boldsymbol{F}_{1}\right)=\left(\sqrt[3]{\frac{\left[1+(\lambda-1)\left(\mu_{1}\right)^{3}\right]^{\kappa}-\left(1-\left(\mu_{1}\right)^{3}\right)^{\kappa}}{\left[1+(\lambda-1)\left(\mu_{1}\right)^{3}\right]^{\kappa}+(\lambda-1)\left(1-\left(\mu_{1}\right)^{3}\right)^{\kappa}}}, \frac{\sqrt[3]{\lambda} \nu_{1}^{\kappa}}{\sqrt[3]{\left[1+(\lambda-1)\left(1-\left(\nu_{1}\right)^{3}\right)\right]^{\kappa}+(\lambda-1)\left(\nu_{1}\right)^{3 \kappa}}}\right)$;

(iv) $\left(\boldsymbol{F}_{1}\right)^{\kappa}=\left(\frac{\sqrt[3]{\lambda}\left(\mu_{1}\right)^{\kappa}}{\sqrt[3]{\left[1+(\lambda-1)\left(1-\left(\mu_{1}\right)^{3}\right)\right]^{\kappa}+(\lambda-1)\left(\mu_{1}^{3}\right)^{\kappa}}}, \sqrt[3]{\frac{\left[1+(\lambda-1)\left(\nu_{1}\right)^{3}\right]^{\kappa}-\left(1-\left(\nu_{1}\right)^{3}\right)^{\kappa}}{\left[1+(\lambda-1)\left(\nu_{1}\right)^{3}\right]^{\kappa}+(\lambda-1)\left(1-\left(\nu_{1}\right)^{3}\right)^{\kappa}}}\right)$.

\subsection{Fermatean fuzzy Hamacher prioritized aggregation operators}

\subsubsection{Fermatean fuzzy Hamacher prioritized arithmetic aggregation operators}

Let us denote by $£$ the set of all non-empty FFNs i.e., $£:=\left\{(\omega, \varpi) \mid(\omega, \varpi) \in[0,1]^{2}, 0 \leq \omega^{3}+\varpi^{3} \leq 1\right\}$ with the partial order $\preceq_{£}$ defined by $\left(\omega_{1}, \varpi_{1}\right) \preceq_{£}\left(\omega_{2}, \varpi_{2}\right) \Longleftrightarrow \omega_{1} \leq \omega_{2}$ and $\varpi_{1} \geq \varpi_{2}$. The top and bottom elements of $£$ are defined by $1_{£}=(1,0)$ and $0_{£}=(0,1)$. Then $£$ becomes a lattice with the partial order $\preceq £$. If $\boldsymbol{F}_{1}$ and $\boldsymbol{F}_{2}$ are two FFNs, then $\boldsymbol{F}_{1} \preceq £ \boldsymbol{F}_{2}$ implies $\boldsymbol{F}_{1} \leq \boldsymbol{F}_{2}$.

The concept of Prioritized Average (PA) was first introduced by Yager [39] in 2008 and was defined as follows:

Definition 3.2. (Yager [39]) Assume that $\mathbb{C}=\left\{\mathbb{C}_{1}, \mathbb{C}_{2}, \ldots, \mathbb{C}_{n}\right\}$ is a collection of criteria with the prioritization among the criteria defined by the linear ordering as $\mathbb{C}_{1} \succ \mathbb{C}_{2} \succ \ldots \succ \mathbb{C}_{n}$, where criteria $\mathbb{C}_{j}$ has a higher priority than $\mathbb{C}_{k}$ for $j<k$ and $n \in \mathbb{N}$. The real number $\mathbb{C}_{j}(x) \in[0,1]$ is the performance of any alternative $x$ under the criteria $\mathbb{C}_{j}$ and

$$
P A\left(\mathbb{C}_{i}\right)=\sum_{j=1}^{n} \Phi_{j} \mathbb{C}_{j}(x)
$$

where $\Phi_{j}=\frac{T_{j}}{\sum_{j=1}^{n} T_{j}}, T_{j}=\prod_{r=1}^{j-1} \mathbb{C}_{k}(x)(j=2, \ldots, n), T_{1}=1$. Then PA is called the prioritized average (PA) operator.

The PA operators are used in the situations where the input arguments are exact values. By combining the Hamacher operations with prioritized inputs based on FF-sets, develop prioritized arithmetic aggregation operators with Fermatean fuzzy numbers based on Hamacher operations. Let $\boldsymbol{F}_{r}=\left(\mu_{r}, \nu_{r}\right)(r=1, \ldots, p)$ be a family of FFEs. We define Fermatean fuzzy Hamacher prioritized arithmetic aggregation operator as follows:

Definition 3.3. The Fermatean fuzzy Hamacher prioritized averaging (FFHPA) operator is a mapping FFHPA: $\boldsymbol{F}^{p} \longrightarrow \boldsymbol{F}$ such that

$$
\begin{aligned}
\text { FFHPA }\left(\boldsymbol{F}_{1}, \boldsymbol{F}_{2}, \ldots, \boldsymbol{F}_{p}\right) & =\oplus_{r=1}^{p}\left(\frac{T_{r}}{\sum_{r=1}^{p} T_{r}} \boldsymbol{F}_{r}\right) \\
& =\frac{T_{1}}{\sum_{r=1}^{p} T_{r}} \boldsymbol{F}_{1} \oplus \frac{T_{2}}{\sum_{r=1}^{p} T_{r}} \boldsymbol{F}_{2} \oplus \ldots \oplus \frac{T_{p}}{\sum_{r=1}^{p} T_{r}} \boldsymbol{F}_{p}
\end{aligned}
$$


where $T_{r}=\prod_{k=1}^{r-1} r t\left(\boldsymbol{F}_{r}\right)(r=2,3, \ldots, p), T_{1}=1$ and $r t\left(\boldsymbol{F}_{r}\right)$ represent the score value of $\boldsymbol{F}_{r}$ $(r=1,2, \ldots, p)$.

In the following theorem we use the mathematical induction and operational rule of FFEs to prove that the aggregation value of a family of FFEs by using FFHPA operator is again an FFE.

Theorem 3.4. Let $\boldsymbol{F}_{r}=\left(\mu_{r}, \nu_{r}\right)(r=1, \ldots, p)$ be a family of FFEs, then the aggregation value of this family by the FFHPA operator is also a FFE, and

$$
\begin{aligned}
& \operatorname{FFHPA}\left(\boldsymbol{F}_{1}, \boldsymbol{F}_{2}, \ldots, \boldsymbol{F}_{p}\right)=\oplus_{r=1}^{p}\left(\frac{T_{r}}{\sum_{r=1}^{p} T_{r}} \boldsymbol{F}_{r}\right) \\
& =\frac{T_{1}}{\sum_{r=1}^{p} T_{r}} \boldsymbol{F}_{1} \oplus \frac{T_{2}}{\sum_{r=1}^{p} T_{r}} \boldsymbol{F}_{2} \oplus \ldots \oplus \frac{T_{p}}{\sum_{r=1}^{p} T_{r}} \boldsymbol{F}_{p}
\end{aligned}
$$

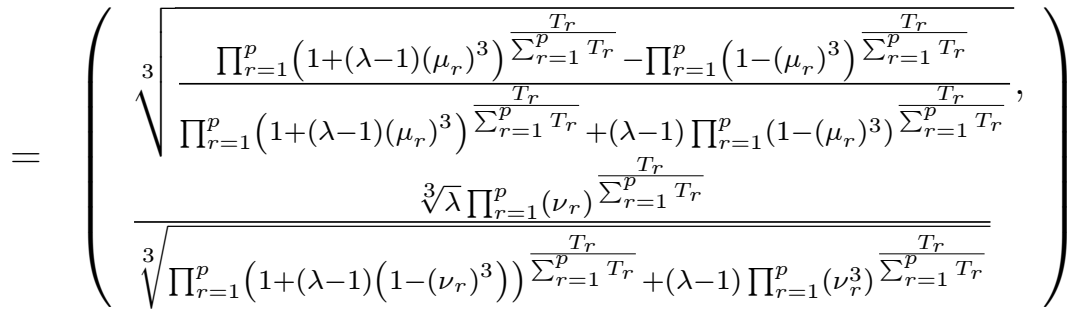

where $T_{r}=\prod_{k=1}^{r-1} r t\left(\boldsymbol{F}_{r}\right)(r=2,3, \ldots, p), T_{1}=1$ and $r t\left(\boldsymbol{F}_{r}\right)$ represent score value of $\boldsymbol{F}_{r}$ $(r=1,2, \ldots, p)$.

Example 1. Let $\boldsymbol{F}_{1}=(0.8,0.5), \boldsymbol{F}_{2}=(0.8,0.7), \boldsymbol{F}_{3}=(0.7,0.8)$ and $\boldsymbol{F}_{4}=(0.6,0.7)$ be four FFEs, $\frac{T_{1}}{\sum_{r=1}^{4} T_{r}}=0.3180 \quad \frac{T_{2}}{\sum_{r=1}^{4} T_{r}}=0.3098 \quad \frac{T_{3}}{\sum_{r=1}^{4} T_{r}}=0.1287 \quad \frac{T_{4}}{\sum_{r=1}^{4} T_{r}}=0.0561$. Suppose $\lambda=3$, then

$$
\begin{aligned}
& \operatorname{FFHPA}\left(\boldsymbol{F}_{1}, \boldsymbol{F}_{2}, \boldsymbol{F}_{3}, \boldsymbol{F}_{4}\right)=\oplus_{r=1}^{4}\left(\frac{T_{r}}{\sum_{r=1}^{4} T_{r}} \boldsymbol{F}_{r}\right)
\end{aligned}
$$

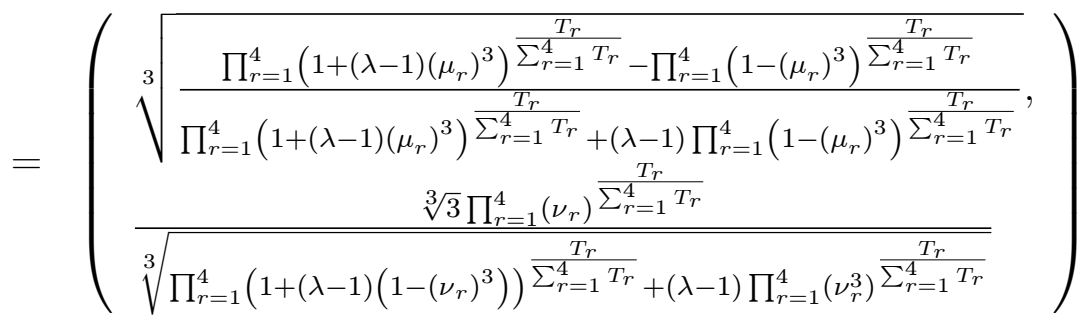

$$
\begin{aligned}
& =(0.7254,0.7424) \text {. }
\end{aligned}
$$

The working parameter in FFHPA operator has two special types which are discussed in the following: 
(1) If $\lambda=1$, then FFHPA is equivalent to the Fermatean fuzzy prioritized average (FFPA) operator:

$$
\begin{aligned}
& \operatorname{FFPA}\left(\boldsymbol{F}_{1}, \boldsymbol{F}_{2}, \ldots, \boldsymbol{F}_{p}\right)=\oplus_{r=1}^{p}\left(\frac{T_{r}}{\sum_{r=1}^{p} T_{r}} \boldsymbol{F}_{r}\right)
\end{aligned}
$$

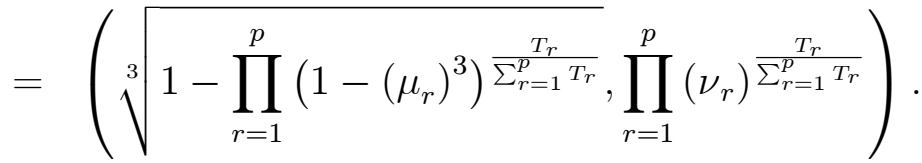

(2) If $\lambda=2$, then FFHPA becomes the Fermatean fuzzy Einstein prioritized average (FFEPA) operator:

$$
\begin{aligned}
& \operatorname{FFEPA}\left(\boldsymbol{F}_{1}, \boldsymbol{F}_{2}, \ldots, \boldsymbol{F}_{p}\right)=\oplus_{r=1}^{p}\left(\frac{T_{r}}{\sum_{r=1}^{p} T_{r}} F_{r}\right)
\end{aligned}
$$

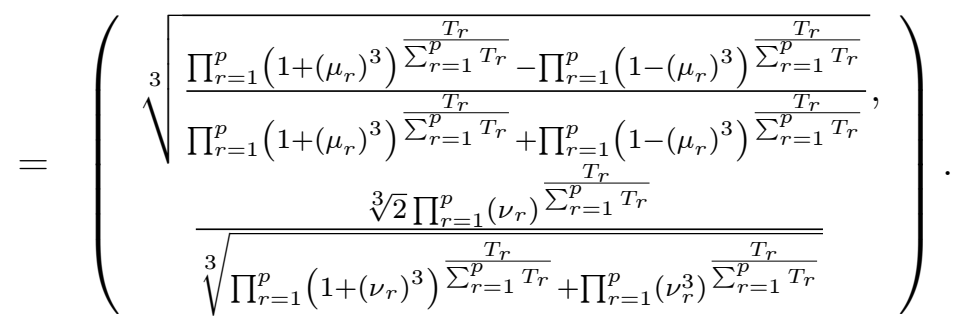

If we consider the weights $\Phi=\left(\Phi_{1}, \Phi_{2}, \ldots, \Phi_{p}\right)^{T}$ of FF-set $\boldsymbol{F}_{r}(r=1,2, \ldots, p)$ such that $\Phi_{r}>$ 0 and $\sum_{r=1}^{p} \Phi_{r}=1$. Then we define FF Hamacher prioritized weighted average (FFHPWA) operator as follows:

Definition 3.5. Let $\boldsymbol{F}_{r}=\left(\mu_{r}, \nu_{r}\right)(r=1, \ldots, p)$ be a family of FFEs. A Fermatean fuzzy Hamacher prioritized weighted average (FFHPWA) operator of a dimension $p$ is a mapping FFHPWA: $\boldsymbol{F}^{p} \longrightarrow \boldsymbol{F}$ with the corresponding weighting vector $\Phi=\left(\Phi_{1}, \Phi_{2}, \ldots, \Phi_{p}\right)^{T}$ such that $\Phi_{r}>0$, and $\sum_{r=1}^{p} \Phi_{r}=1$. Then

$$
F F H P W A_{\Phi}\left(\boldsymbol{F}_{1}, \boldsymbol{F}_{2}, \ldots, \boldsymbol{F}_{p}\right)=\oplus_{r=1}^{p}\left(\frac{\Phi_{r} T_{r}}{\sum_{r=1}^{p} \Phi_{r} T_{r}} \boldsymbol{F}_{r}\right)
$$

where $T_{r}=\prod_{k=1}^{r-1} r t\left(\boldsymbol{F}_{r}\right)(r=2,3, \ldots, p), T_{1}=1$ and $r t\left(\boldsymbol{F}_{r}\right)$ represent the rating value of $\boldsymbol{F}_{r}$ $(r=1,2, \ldots, p)$

As a consequence of Definition 3.5, we have the theorem stated below.

Theorem 3.6. A Fermatean fuzzy Hamacher prioritized weighted average (FFHPWA) oper- 
ator of a dimension $p$ returns a FF-set and

$$
\begin{aligned}
& F F H P W A_{\Phi}\left(\boldsymbol{F}_{1}, \boldsymbol{F}_{2}, \ldots, \boldsymbol{F}_{p}\right)=\oplus_{r=1}^{p}\left(\frac{\Phi_{r} T_{r}}{\sum_{r=1}^{p} \Phi_{r} T_{r}} \boldsymbol{F}_{r}\right)
\end{aligned}
$$

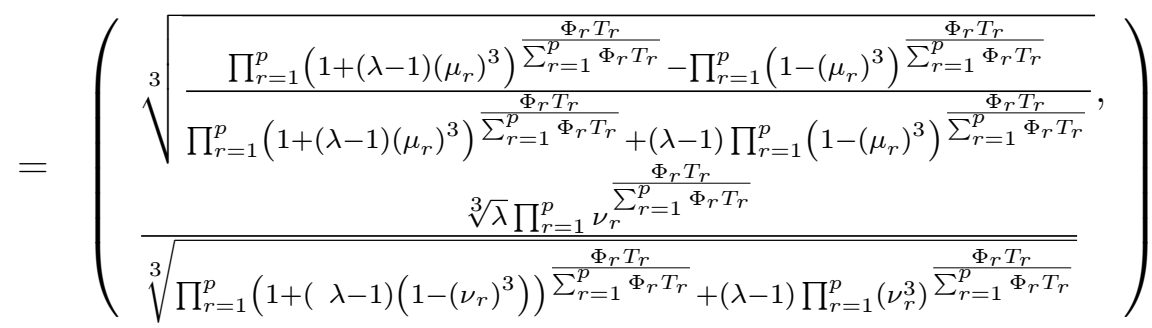

where where $T_{r}=\prod_{k=1}^{r-1} r t\left(\boldsymbol{F}_{r}\right)(r=2,3, \ldots, p), T_{1}=1$ and $r t\left(\boldsymbol{F}_{r}\right)$ represent the rating value of $\boldsymbol{F}_{r}(r=1,2, \ldots, p)$ and $\Phi=\left(\Phi_{1}, \Phi_{2}, \ldots, \Phi_{p}\right)^{T}$ is the weight vector such that $\Phi_{r}>0$, and $\sum_{r=1}^{p} \Phi_{r}=1$. $\lambda$.

In the following we discuss two special case of FFHPWA operator for the working parameter

(1) For $\lambda=1$, the $(F F H P W A)$ reduces to Fermatean fuzzy prioritized weighted average (FFPWA) operator:

$$
\begin{aligned}
& F F P W A_{\Phi}\left(\boldsymbol{F}_{1}, \boldsymbol{F}_{2}, \ldots, \boldsymbol{F}_{p}\right)=\otimes_{r=1}^{p}\left(\frac{\Phi_{r} T_{r}}{\sum_{r=1}^{p} \Phi_{r} T_{r}} \boldsymbol{F}_{r}\right) \\
& =\left(\sqrt[3]{1-\prod_{r=1}^{p}\left(1-\left(\mu_{r}\right)^{3}\right)^{\frac{\Phi_{r} T_{r}}{\sum_{r=1}^{p}}}}, \prod_{r=1}^{p}\left(\nu_{r}\right)^{\frac{\Phi_{r} T_{r}}{\sum_{r=1}^{p} \Phi_{r} T_{r}}}\right) .
\end{aligned}
$$

(2) For $\lambda=2$, FFHPWA reduces to Fermatean fuzzy Einstein prioritized weighted average (FFEPWA) operator:

$$
\begin{aligned}
& F F E P W A_{\Phi}\left(\boldsymbol{F}_{1}, \boldsymbol{F}_{2}, \ldots, \boldsymbol{F}_{p}\right)=\oplus_{r=1}^{p}\left(\frac{\Phi_{r} T_{r}}{\sum_{r=1}^{p} \Phi_{r} T_{r}} \boldsymbol{F}_{r}\right)
\end{aligned}
$$

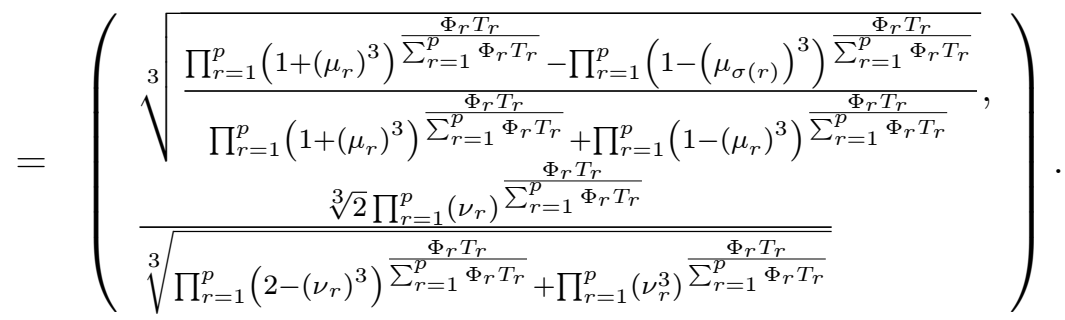

Example 2. Let $\boldsymbol{F}_{1}=(0.6,0.4), \boldsymbol{F}_{2}=(0.7,0.6), \quad \boldsymbol{F}_{3}=(0.8,0.5)$, and $\boldsymbol{F}_{4}=(0.5,0.8)$ be four FFEs and $\Phi=(0.2,0.1,0.3,0.4)^{T}$ be the weighting vector. Then 
$\frac{\Phi_{1} T_{1}}{\sum_{r=1}^{4} \Phi_{r} T_{r}}=0.474$

$\frac{\Phi_{2} T_{2}}{\sum_{r=1}^{4} \Phi_{r} T_{r}}=0.1336$

$\frac{\Phi_{3} T_{3}}{\sum_{r=1}^{4} \Phi_{r} T_{r}}=0.2781$

$\frac{\Phi_{4} T_{4}}{\sum_{r=1}^{4} \Phi_{r} T_{r}}=$

0.1134 and the aggregation value of FFEs for $(\lambda=3)$ and by using definition of FFHPWA operator we get

$$
\begin{aligned}
& C F H P W A_{\Phi}\left(\boldsymbol{F}_{1}, \boldsymbol{F}_{2}, \boldsymbol{F}_{3}, \boldsymbol{F}_{4}\right)=\oplus_{r=1}^{4}\left(\frac{\Phi_{r} T_{r}}{\sum_{r=1}^{4} \Phi_{r} T_{r}} \boldsymbol{F}_{r}\right)
\end{aligned}
$$

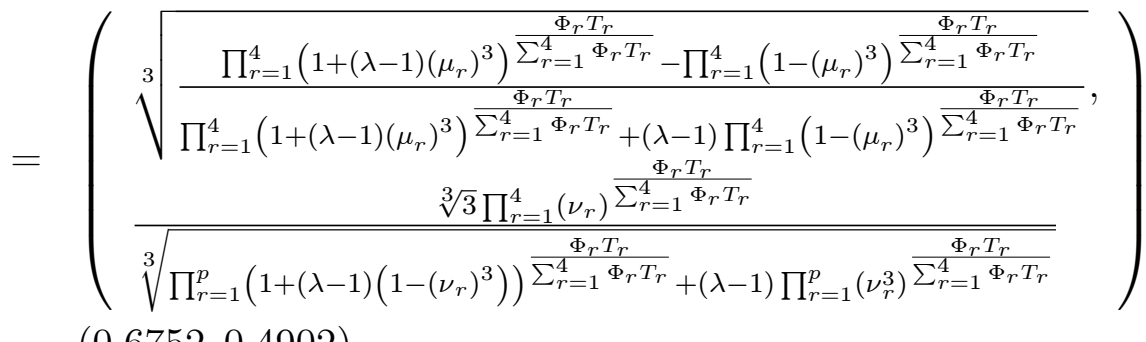

$$
\begin{aligned}
& =(0.6752,0.4902) \text {. }
\end{aligned}
$$

\section{Fermatean fuzzy Hamacher prioritized geometric ag- gregation operators}

Let $\boldsymbol{F}_{r}=\left(\mu_{r}, \nu_{r}\right)(r=1, \ldots, p)$ be a family of FFEs. We define Fermatean fuzzy Hamacher prioritized geometric aggregation operator as follows:

Definition 4.1. The Fermatean fuzzy Hamacher prioritized averaging (FFHPG) operator is a mapping FFHPG: $\boldsymbol{F}^{p} \longrightarrow \boldsymbol{F}$ such that

$$
\begin{aligned}
\text { FFHPA }\left(\boldsymbol{F}_{1}, \boldsymbol{F}_{2}, \ldots, \boldsymbol{F}_{p}\right) & =\otimes_{r=1}^{p}\left(\boldsymbol{F}_{r}\right)^{\frac{T_{r}}{\sum_{r=1}^{p} T_{r}}} \\
& =\left(\boldsymbol{F}_{1}\right)^{\frac{T_{1}}{\sum_{r=1}^{p} T_{r}}} \otimes\left(\boldsymbol{F}_{2}\right)^{\frac{T_{2}}{\sum_{r=1}^{p} T_{r}}} \otimes \ldots \otimes\left(\boldsymbol{F}_{p}\right)^{\frac{T_{p}}{\sum_{r=1}^{p} T_{r}}}
\end{aligned}
$$

where $T_{r}=\prod_{k=1}^{r-1} r t\left(\boldsymbol{F}_{r}\right)(r=2,3, \ldots, p), T_{1}=1$ and $r t\left(\boldsymbol{F}_{r}\right)$ represent score value of $\boldsymbol{F}_{r}$ $(r=1,2, \ldots, p)$.

In the following theorem we use the mathematical induction and operational rule of FFEs to prove that the aggregation value of a family of FFEs by using FFHPG operator is again an FFE.

Theorem 4.2. Let $\boldsymbol{F}_{r}=\left(\mu_{r}, \nu_{r}\right)(r=1, \ldots, p)$ be a family of FFEs, then the aggregation value 
of this family by the FFHPG operator is also a FFE, and

$$
\begin{aligned}
& F F H P G\left(\boldsymbol{F}_{1}, \boldsymbol{F}_{2}, \ldots, \boldsymbol{F}_{p}\right)=\otimes_{r=1}^{p}\left(\boldsymbol{F}_{r}\right)^{\frac{T_{r}}{\sum_{r=1}^{p} T_{r}}} \\
& =\left(\boldsymbol{F}_{1}\right)^{\frac{T_{1}}{\sum_{r=1}^{p} T_{r}}} \otimes\left(\boldsymbol{F}_{2}\right)^{\frac{T_{2}}{\sum_{r=1}^{p} T_{r}}} \otimes \ldots \otimes\left(\boldsymbol{F}_{p}\right)^{\frac{T_{p}}{\sum_{r=1}^{p} T_{r}}}
\end{aligned}
$$

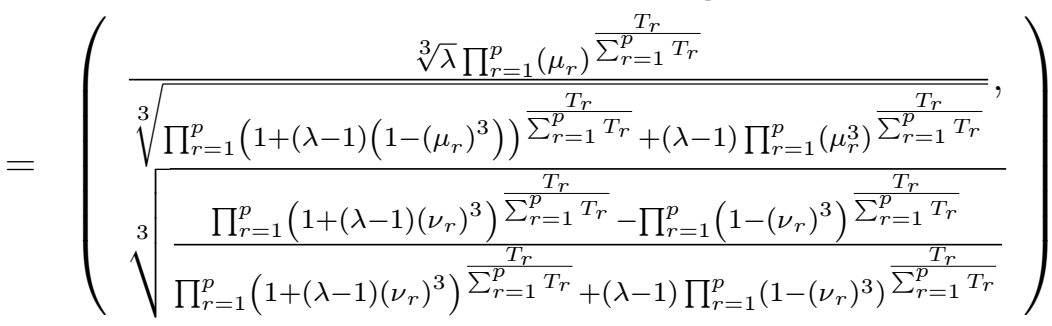

where $T_{r}=\prod_{k=1}^{r-1} r t\left(\boldsymbol{F}_{r}\right)(r=2,3, \ldots, p), T_{1}=1$ and $r t\left(\boldsymbol{F}_{r}\right)$ represent score value of $\boldsymbol{F}_{r}$ $(r=1,2, \ldots, p)$.

Example 3. Let $\boldsymbol{F}_{1}=(0.8,0.5), \boldsymbol{F}_{2}=(0.8,0.7), \boldsymbol{F}_{3}=(0.7,0.8)$ and $\boldsymbol{F}_{4}=(0.6,0.7)$ be four FFEs, $\frac{T_{1}}{\sum_{r=1}^{4} T_{r}}=0.3180 \quad \frac{T_{2}}{\sum_{r=1}^{4} T_{r}}=0.3098 \quad \frac{T_{3}}{\sum_{r=1}^{4} T_{r}}=0.1287 \quad \frac{T_{4}}{\sum_{r=1}^{4} T_{r}}=0.0561$. Suppose $\lambda=3$, then

$$
\begin{aligned}
& \operatorname{FFHPA}\left(\boldsymbol{F}_{1}, \boldsymbol{F}_{2}, \boldsymbol{F}_{3}, \boldsymbol{F}_{4}\right)=\otimes_{r=1}^{4}\left(\boldsymbol{F}_{r}\right)^{\frac{T_{r}}{\sum_{r=1}^{4} T_{r}}} \\
& =\left(\begin{array}{l}
\frac{\sqrt[3]{3} \prod_{r=1}^{4}\left(\mu_{r}\right)^{\frac{T_{r}}{\sum_{r=1} T_{r}}}}{\sqrt[3]{\prod_{r=1}^{4}\left(1+(\lambda-1)\left(1-\left(\mu_{r}\right)^{3}\right)\right)^{{\overline{T_{r}}}_{r=1}^{4}}+(\lambda-1) \prod_{r=1}^{4}\left(\mu_{r}^{3}\right)^{\frac{\sum_{r=1}^{4}}{T_{r}}}}} \\
\sqrt[3]{\frac{\prod_{r=1}^{4}\left(1+(\lambda-1)\left(\nu_{r}\right)^{3}\right)^{\frac{T_{r}}{\sum_{r=1}^{4} T_{r}}}-\prod_{r=1}^{4}\left(1-\left(\nu_{r}\right)^{3}\right)^{\frac{T_{r}}{\sum_{r=1}^{4} T_{r}}}}{\prod_{r=1}^{4}\left(1+(\lambda-1)\left(\nu_{r}\right)^{3}\right)^{\bar{T}_{r=1}^{4}}}}
\end{array}\right) \\
& =(0.8886,0.6132) \text {. }
\end{aligned}
$$

Further, we offer two special cases of FFHPG operator:

(1) For $\lambda=1$, FFHPG becomes the Fermatean fuzzy prioritized geometric (FFPG) operator:

$$
\begin{aligned}
\text { FFPG }\left(\boldsymbol{F}_{1}, \boldsymbol{F}_{2}, \ldots, \boldsymbol{F}_{p}\right) & =\otimes_{r=1}^{p}\left(\boldsymbol{F}_{r}\right)^{\sum_{r=1}^{T_{r}}} \\
& =\left(\prod_{r=1}^{p}\left(\mu_{r}\right)^{\frac{T_{r}}{\sum_{r=1}^{r} T_{r}}}, \sqrt[3]{1-\prod_{r=1}^{p}\left(1-\left(\nu_{r}\right)^{3}\right)^{\frac{T_{r}^{r}}{\sum_{r=1}^{T_{r}}}}}\right)
\end{aligned}
$$

(2) For $\lambda=2$, FFHPG reduces to Fermatean fuzzy Einstein prioritized geometric (FFEPG) 
operator:

$$
\begin{aligned}
\text { FFEPG } & \left(\boldsymbol{F}_{1}, \boldsymbol{F}_{2}, \ldots, \boldsymbol{F}_{p}\right)=\otimes_{r=1}^{p}\left(\boldsymbol{F}_{r}\right)^{\frac{T_{r}}{\sum_{r=1}^{r} T_{r}}} \\
= & \left(\frac{\sqrt[3]{2} \prod_{r=1}^{p}\left(\mu_{r}\right)^{\frac{T_{r}^{r}}{\sum_{r=1}^{T_{r}}}}}{\sqrt[3]{\prod_{r=1}^{p}\left(2-\left(\mu_{r}\right)^{3}\right)^{\sum_{r=1}^{T_{r}} T_{r}}}+\prod_{r=1}^{p}} \sqrt[3]{\frac{\prod_{r=1}^{p}\left(1-\left(\nu_{r}\right)^{3}\right)^{\frac{T_{r}}{\sum_{r=1}^{T_{r}}}}-\prod_{r=1}^{p}\left(1-\left(\nu_{r}\right)^{3}\right)^{\frac{T_{r}}{\sum_{r=1}^{T_{r}}}}}{\prod_{r=1}^{p}\left(1-\left(\lambda_{r}\right)^{3}\right)^{\sum_{r=1}^{T_{r}}}}}\right)
\end{aligned}
$$

In the following we introduce FFHPWG operator.

Definition 4.3. Let $\boldsymbol{F}_{r}=\left(\mu_{r}, \nu_{r}\right)(r=1,2, \ldots, p)$ be a family of FFEs. The FFHPWG is a mapping from $F^{p}$ to $F$ such that

$$
F F H P W G_{\Phi}\left(\boldsymbol{F}_{1}, \boldsymbol{F}_{2}, \ldots, \boldsymbol{F}_{p}\right)=\otimes_{r=1}^{p}\left(\boldsymbol{F}_{r}\right)^{\frac{\Phi_{r} T_{r}}{\sum_{r=1}^{r} \Phi_{r} T_{r}}}
$$

where $T_{r}=\prod_{k=1}^{r-1} r t\left(\boldsymbol{F}_{r}\right)(r=2,3, \ldots, p), T_{1}=1$ and $r t\left(\boldsymbol{F}_{r}\right)$ represent the score value of $\boldsymbol{F}_{r}$ and $\Phi=\left(\Phi_{1}, \Phi_{2}, \ldots, \Phi_{p}\right)^{T}$ is the weighting vector of $\boldsymbol{F}_{r}(r=1,2, \ldots, p)$ such that $\Phi_{r}>0$ and $\sum_{r=1}^{p} \Phi_{r}=1$.

Using FFHPWG operator, and the operational rules of Definition 3.1, we can prove the following subsequent theorem easily.

Theorem 4.4. Let $\boldsymbol{F}_{r}=\left(\mu_{r}, \nu_{r}\right)(r=1,2, \ldots, p)$ be a family of FFEs. The FFHPWG operator is a mapping $F^{p} \longrightarrow F$ such that

$$
\begin{aligned}
& F F H P W G_{\Phi}\left(\boldsymbol{F}_{1}, \boldsymbol{F}_{2}, \ldots, \boldsymbol{F}_{p}\right)=\otimes_{r=1}^{p}\left(\boldsymbol{F}_{r}\right)^{\frac{\Phi_{r} T_{r}}{\sum_{r=1}^{r} \Phi_{r} T_{r}}}
\end{aligned}
$$

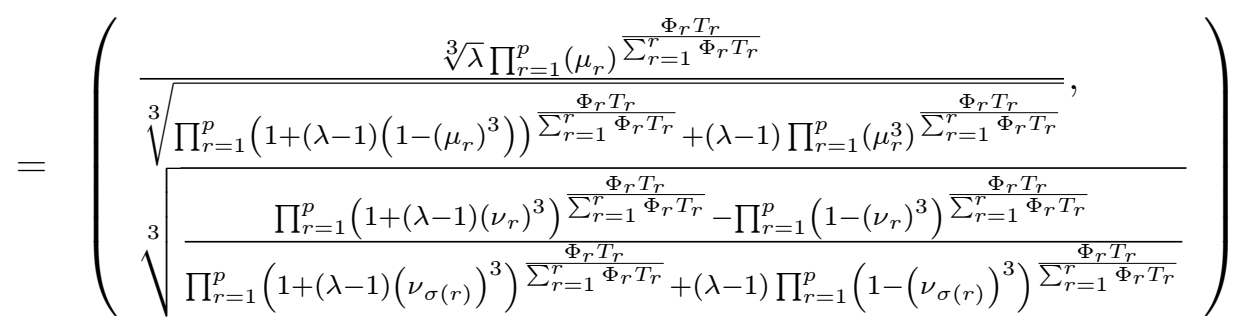

where $T_{r}=\prod_{k=1}^{r-1} r t\left(\boldsymbol{F}_{r}\right)(r=2,3, \ldots, p), T_{1}=1$ and $r t\left(\boldsymbol{F}_{r}\right)$ represent score value of $\boldsymbol{F}_{r}$ $(r=1,2, \ldots, p)$ and $\Phi=\left(\Phi_{1}, \Phi_{2}, \ldots, \Phi_{p}\right)^{T}$ is the weighting vector of $\boldsymbol{F}_{r}(r=1,2, \ldots, p)$ such that $\Phi_{r}>0$ and $\sum_{r=1}^{p} \Phi_{r}=1$.

We investigate two special cases of FFHPWG operator in the following:

(1) For $\lambda=1$, FFHPWG minimizes to Fermatean fuzzy prioritized weighted geometric 
(FFPWG) operator:

$$
\begin{aligned}
F F P W G_{\Phi}\left(\boldsymbol{F}_{1}, \boldsymbol{F}_{2}, \ldots, \boldsymbol{F}_{r}\right) & =\otimes_{r=1}^{p}\left(\boldsymbol{F}_{r}\right)^{\frac{\Phi_{r} T_{r} T_{r}}{\sum_{r=1} \Phi_{r} T_{r}}} \\
& =\left(\prod_{r=1}^{p}\left(\mu_{r}\right)^{\frac{\Phi_{r} T_{r}}{\sum_{r=1}^{\Phi_{r} T_{r}}}}, \sqrt[3]{1-\prod_{r=1}^{p}\left(1-\left(\nu_{r}\right)^{3}\right)^{\frac{\Phi_{r} T_{r}}{\sum_{r} T_{r} T_{r}}}}\right) .
\end{aligned}
$$

(2) For $\lambda=2$, FFHPWG minimizes to Fermatean fuzzy Einstein prioritized weighted geometric (FFEPWG) operator:

$$
\begin{aligned}
& F F E P W G_{\Phi}\left(\boldsymbol{F}_{1}, \boldsymbol{F}_{2}, \ldots, \boldsymbol{F}_{r}\right)=\otimes_{r=1}^{p}\left(\boldsymbol{F}_{r}\right)^{\frac{\Phi_{r} T_{r}}{\sum_{r=1}^{r} \Phi_{r} T_{r}}}
\end{aligned}
$$

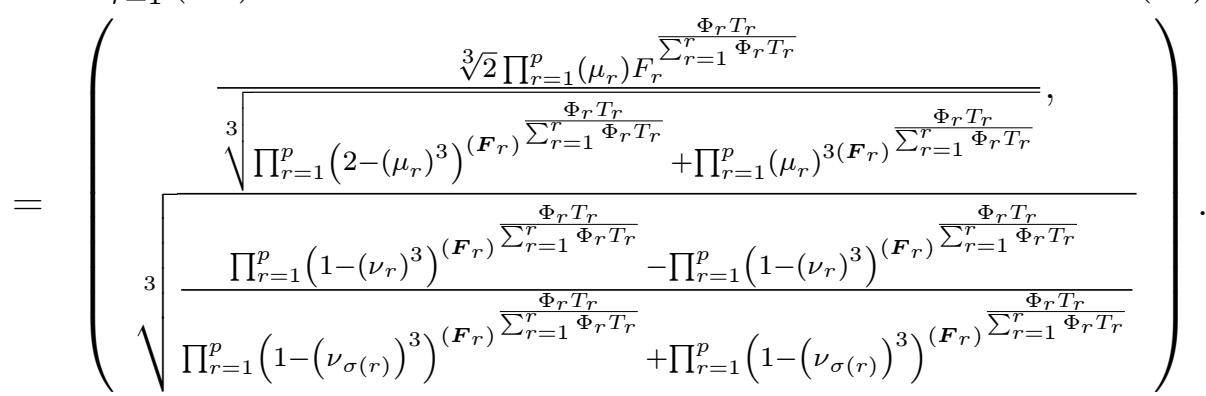

\section{Fuzzy Modeling of MADM: The case of Fermatean Fuzzy Information}

We shall apply FF-Dombi prioritized AOs constructed in the previous sections to solve a MADM problem with FF information. Denote a discrĕte set of alternatives by $\mathbb{A}=$ $\left\{\boldsymbol{A}_{1}, \boldsymbol{A}_{2}, \ldots, \boldsymbol{A}_{m}\right\}$, we also denote by $\mathbb{G}=\left\{\boldsymbol{G}_{1}, \boldsymbol{G}_{2}, \ldots, \boldsymbol{G}_{r}\right\}$, the set of attributes, we assume that there is a prioritization among these attributes and let the prioritization be a linear ordering $\boldsymbol{G}_{1} \succ \boldsymbol{G}_{2} \succ \ldots \succ \boldsymbol{G}_{r}$ indicating that the attribute $\boldsymbol{G}_{\zeta}$ has a higher priority than $\boldsymbol{G}_{\xi}$ if $\zeta<\xi$. Let $\varnothing=\left\{\varnothing_{1}, \varnothing_{2}, \ldots, \varnothing_{r}\right\}$ be the weight vector for the attributes $\boldsymbol{G}_{\xi}(\xi=1,2,3, \ldots, r)$ such that $\varnothing_{\xi}>0$ and $\sum_{\xi=1}^{r} \varnothing_{\xi}=1$. Suppose that $M=\left(\boldsymbol{F}_{\phi \xi}\right)_{m \times n}=\left(\mu_{\dot{\phi} \xi}, \delta_{\dot{\phi} \xi}\right)_{m \times n}$ is the FF decision matrix, where $\mu_{\dot{\phi} \xi}$ represents the degree of membership, that the alternative $\boldsymbol{A}_{\xi} \in \mathbb{A}$ satisfies the alternative $\boldsymbol{G}_{\xi}$ and $\delta_{\phi \xi}$ denotes the degree of non-mebmberrship that the alternative $\boldsymbol{A}_{\xi} \in \mathbb{A}$ does not satisfies the attribute $\boldsymbol{G}_{\xi}$ considered by the decision makers such that $\mu_{\dot{\phi} \xi}^{3}+\delta_{\phi \xi}^{3} \leq 1$ and $\mu_{\phi \xi}, \delta_{\phi \xi} \subset[0,1],(\phi=1,2, \ldots, m)$ and $(\xi=1,2, \ldots, n)$ that the DMs proposed for the attributes $\boldsymbol{G}_{\xi}$. To follow the above discussion we utilize the methods developed in previous section and design an algorithm to solve multiple attribute decision-making problem based on FF-environment.

\section{Algorithm}


Step 1. Calculate the value of $T_{\phi \xi}(\phi=1,2, \ldots, m ; \xi=1,2, \ldots, r)$ using the formula as follows by

$$
T_{\phi \xi}=\prod_{k=1}^{\xi-1} \operatorname{Score}\left(\boldsymbol{F}_{\phi k}\right)(\phi=1,2, \ldots, m ; \xi=1,2, \ldots, r ; k=2,3, \ldots, m)
$$

and

$$
T_{\phi 1}=1,(\phi=1,2, \ldots, m) .
$$

Step 2. Apply the operator FFHPWA on the decision matrix $M$ where

$$
\begin{aligned}
& \boldsymbol{F}_{\phi}=F F H P W A\left(\boldsymbol{F}_{\phi 1}, \boldsymbol{F}_{\phi_{2}}, \ldots, \boldsymbol{F}_{\phi_{r}}\right) \\
& =\bigoplus_{\xi=1}^{r}\left(\frac{\varnothing_{\xi} T_{\dot{\phi} \xi}}{\sum_{\xi=1}^{r} \varnothing_{\xi} T_{\phi \xi}} \boldsymbol{F}_{\phi \xi}\right) \\
& =\frac{\varnothing_{1} T_{\phi 1}}{\sum_{\xi=1}^{r} \varnothing_{\xi} T_{\phi \xi}} \boldsymbol{F}_{1} \oplus \frac{\varnothing_{2} T_{\dot{\phi} 2}}{\sum_{\xi=1}^{r} \varnothing_{\xi} T_{\phi \xi}} \boldsymbol{F}_{2} \oplus \ldots \oplus \frac{\varnothing_{r} T_{\phi r}}{\sum_{\xi=1}^{r} \varnothing_{\xi} T_{\phi \xi}} \boldsymbol{F}_{r}
\end{aligned}
$$

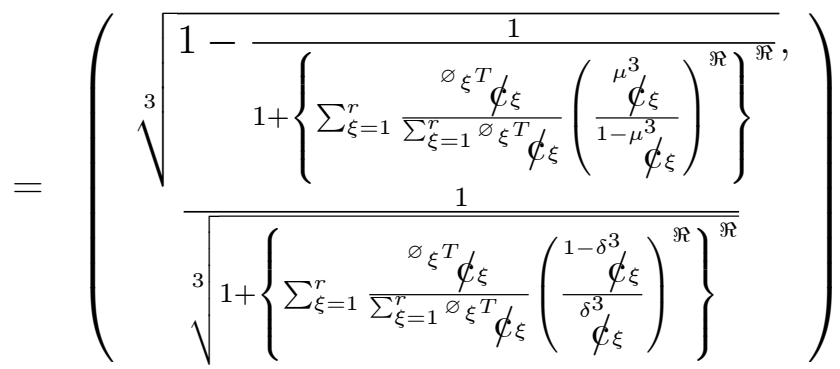

or apply FFHPWG operator

$$
\begin{aligned}
& \boldsymbol{F}_{\phi}=F F H P W G\left(\boldsymbol{F}_{\phi 1}, \boldsymbol{F}_{\phi 2}, \ldots, \boldsymbol{F}_{\phi r}\right) \\
& =\bigotimes_{\xi=1}^{r}\left(\boldsymbol{F}_{\phi \xi}\right)^{\frac{{ }_{\sigma_{\xi}^{T}} \phi_{\xi}^{r}}{\sum_{\xi=1}^{r} \xi^{T} \phi \xi}}
\end{aligned}
$$

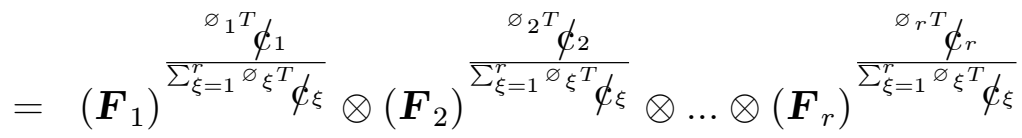

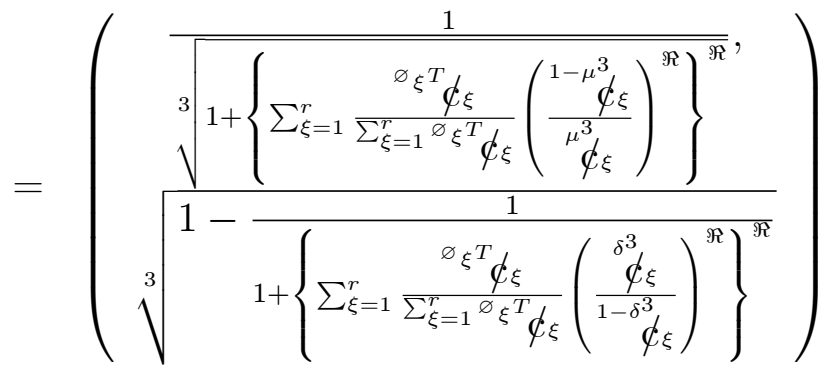


to get the aggregated values of $\boldsymbol{F}_{\phi}(\phi=1,2, \ldots, m)$ of the alternatives $\boldsymbol{A}_{\phi}$.

Step 3. Calculate the values of the score function $\operatorname{Score}\left(\boldsymbol{F}_{\phi}\right)(\phi=1,2, \ldots, m)$ of all the aggregated FFNs $\boldsymbol{F}_{\phi}(\phi=1,2, \ldots, m)$ obtained in Step 2. If the value of score functions Score $\left(\boldsymbol{F}_{\phi}\right)$ and Score $\left(\boldsymbol{F}_{\xi}\right)$ are not different then apply the accuracy function acc $\left(\boldsymbol{F}_{\phi}\right)$ and $\operatorname{acc}\left(\boldsymbol{F}_{\xi}\right)$ for the ranking order of alternatives $\boldsymbol{A}_{\phi}(\phi=1,2, \ldots, m)$.

Step 5. End

\section{Experimental example and comparative discussion}

\subsection{Example description}

We discuss the selection process of teaching staff of our university. To promote the education system of Abdul Wali Khan University, the Department of Mathematics want to recruit overseas outstanding educationists. After some important meetings in the Department an expert team is selected to complete the process of selection of outstanding teachers. The panel of experts consists on university vice chancellor (VC), dean of physical and numerical sciences (P\&NS) and human resource development officer. This team of expert will analyses a set of five candidates $\boldsymbol{A}_{\phi}(\phi=1,2,3,4,5)$ following the four attributes $\boldsymbol{G} 1$ : Qualification, $\boldsymbol{G} 2$ : Teaching ability, G3: Research expertise and G4: Quality research publications. University VC has absolute priority in decision-making, dean of P\&NS comes next. Further they will be strict in their principle of combine ability and will not influence by any political integrity. The prioritization criteria is defined as $\mathbf{G} \mathbf{1} \succ \mathbf{G} \mathbf{2} \succ \mathbf{G} \mathbf{3} \succ \mathbf{G} 4$, where the symbol $\succ$ is used to represents prefer than relation. The team will use FFNs in the evaluation of candidates $\boldsymbol{A}_{\phi}$ $(\phi=1,2,3,4,5)$. The attribute weight vector in the selection process is $\varnothing=(0.2,0.2,0.3,0.3)^{T}$ and the decision matrix for this model is $\mathbb{F}=\left(\mathcal{F}_{\phi \xi}\right)_{4 \times 5}$ which is represented in Table 1 , where $\mathcal{F}_{\phi \xi}$ are FFNs.

Table 1. Fermatean fuzzy decision matrix

\begin{tabular}{llllll}
\hline & $\boldsymbol{A}_{1}$ & $\boldsymbol{A}_{2}$ & $\boldsymbol{A}_{3}$ & $\boldsymbol{A}_{4}$ & $\boldsymbol{A}_{5}$ \\
\hline $\mathbf{G}_{1}$ & $(.4, .6)$ & $(.8, .2)$ & $(.5, .5)$ & $(.7, .3)$ & $(.4, .8)$ \\
\hline $\boldsymbol{G}_{2}$ & $(.8, .6)$ & $(.7, .4)$ & $(.4, .5)$ & $(.5, .8)$ & $(.5, .6)$ \\
\hline $\mathbf{G}_{3}$ & $(.8, .1)$ & $(.7, .5)$ & $(.6, .4)$ & $(.9, .6)$ & $(.3, .9)$ \\
\hline $\mathbf{G}_{4}$ & $(.8, .3)$ & $(.5, .3)$ & $(.7, .2)$ & $(.6, .7)$ & $(.3, .7)$ \\
\hline
\end{tabular}

In order to select the most desirable candidate $\boldsymbol{A}_{\phi}(\phi=1,2,3,4,5)$, we apply FFHPWA and FFHPWG operators in the following Steps of algorithm 


\subsubsection{FFHPWA operator}

Step 1. Apply Equs. (7) and (8) to compute the values of $T_{\phi \xi}(\phi=1,2, \ldots, m ; \xi=1,2, \ldots, r)$ which are given in the following matrix

$$
T_{\phi \xi}=\left[\begin{array}{lllll}
1.000 & .4240 & .3188 & .1594 & .1048 \\
1.000 & .6480 & .4143 & .1945 & .0596 \\
1.000 & .7555 & .4600 & .2649 & .2003 \\
1.000 & .7425 & .4076 & .2720 & .1187
\end{array}\right]
$$

Step 2. For $\lambda=1$, we apply FFHPWA operator to aggregate the over all preference values of FFNs $\mathcal{F}_{\phi}$ of the candidates $\boldsymbol{A}_{\phi}(\phi=1,2,3,4,5)$, we get

$$
\mathcal{F}_{1}=(0.8389,0.1640), \mathcal{F}_{2}=(0.7027,0.3188), \mathcal{F}_{3}=(0.5097,0.5226), \mathcal{F}_{4}=(0.5654,0.8165) \text {, }
$$
$\mathcal{F}_{5}=(0.2163,0.9519)$.

Step 3. Calculate the score value using score $\left(\mathcal{F}_{\phi}\right)$ function of the FFNs $\mathcal{F}_{\phi}(\phi=1,2,3,4,5)$, we get:

$\operatorname{score}\left(\mathcal{F}_{1}\right)=0.7929, \operatorname{score}\left(\mathcal{F}_{2}\right)=0.6572, \operatorname{score}\left(\mathcal{F}_{3}\right)=0.4948, \operatorname{score}\left(\mathcal{F}_{4}\right)=0.3182, \operatorname{score}\left(\mathcal{F}_{5}\right)=$ 0.0737 .

Step 4. Rank all the candidates $\boldsymbol{A}_{\phi}(\phi=1,2,3,4,5)$, according to the values of score function score $\left(\mathcal{F}_{\phi}\right)(\phi=1,2,3,4,5)$, of the candidates as $\boldsymbol{A}_{1}>\boldsymbol{A}_{2}>\boldsymbol{A}_{3}>\boldsymbol{A}_{4}>\boldsymbol{A}_{5}$.

Step 5. $\boldsymbol{A}_{1}$ is selected as the best candidate.

\subsubsection{FFHPWG operator}

If we utilize the FFHPWG operator, then the procedure is similar as above

Step 1. For $\lambda=1$, we apply FFHPWG operator to aggregate the over all preference values of FFNs $\mathcal{F}_{\phi}$ of the candidates $\boldsymbol{A}_{\phi}(\phi=1,2,3,4,5)$, we get

$$
\mathcal{F}_{1}=(0.4848,0.5956), \mathcal{F}_{2}=(0.6377,0.3993), \mathcal{F}_{3}=(0.6580,0.3826), \mathcal{F}_{4}=(0.8594,0.4974) \text {, }
$$
$\mathcal{F}_{5}=(0.8094,0.5293)$.

Step 2. Calculate the score value using score $\left(\mathcal{F}_{\phi}\right)$ function of the FFNs $\mathcal{F}_{\phi}(\phi=1,2,3,4,5)$, we get:

$\operatorname{score}\left(\mathcal{F}_{1}\right)=0.4513, \operatorname{score}\left(\mathcal{F}_{2}\right)=0.5978, \operatorname{score}\left(\mathcal{F}_{3}\right)=0.6144, \operatorname{score}\left(\mathcal{F}_{4}\right)=0.7558, \operatorname{score}\left(\mathcal{F}_{5}\right)=$ 0.6909 .

Step 3. Rank all the candidates $\boldsymbol{A}_{\phi}(\phi=1,2,3,4,5)$, according to the values of score function, score $\left(\mathcal{F}_{\phi}\right)(\phi=1,2,3,4,5)$, of the candidates as $\boldsymbol{A}_{4}>\boldsymbol{A}_{5}>\boldsymbol{A}_{3}>\boldsymbol{A}_{2}>\boldsymbol{A}_{1}$.

Step 4. $\boldsymbol{A}_{4}$ is selected as the best candidate for the post. 
From above discussion, we observe that the overall ranking orders of all the candidates are different by utilizing the two operators, in FFHPWA operator $\boldsymbol{A}_{1}$ is the most suitable candidate for the post while by applying FFHPWG operator, the most desirable candidate is $\boldsymbol{A}_{4}$.

\subsection{Comparison of proposed and existing operators}

From Table 2, we observe that the ranking order of alternative by using different methods are different. But the best alternatives of the same type of aggregation operator are same, e.g., the best alternative in FFWA, FFWG, FFWPA and FFWPG operators is $A_{3}$. The best alternatives for the methods FFHWA operator is $A_{5}$ and for FFHWG operator is $A_{4}$. This means that the FFHWA and FFHWG operators have a rare fluctuations in ranking order. FFHOWA and FFHOWG operators have same ranking for best alternative i.e., $A_{5}$. The other methods FFHPA, FFHPG, FFHPWA and FFHPWG operators have similar fluctuations in the ranking order as in case of FFHWA and FFHWG operators.

Table 2. Proposed and existing aggregation methods

\begin{tabular}{|c|c|c|c|c|c|c|}
\hline Methods & $S\left(\mathcal{F}_{1}\right)$ & \multicolumn{2}{|c|}{$S\left(\mathcal{F}_{2}\right)$} & & & Ranking order \\
\hline$F F W A$ & -1.305 & -1.175 & -0.429 & -1.477 & -0.498 & $A_{3} \succ A_{5} \succ$ \\
\hline$F F W G$ & -1.271 & -1.176 & -0.406 & 1.351 & -1.181 & \\
\hline$F F W P A$ & -0.799 & -0.879 & -0.376 & -1.237 & -0.736 & $A_{3}$ \\
\hline$F F W P G$ & -0.533 & -0.705 & -0.338 & -1.080 & -0.586 & $A_{3}$ \\
\hline FFHWA & 0.0996 & 0.2004 & 0.0110 & 0.1646 & 0.2061 & $A_{2} \succ$ \\
\hline$F F H$ & -0.0472 & 0.0904 & -0.0008 & 0.1497 & 427 & $A_{5} \succ$ \\
\hline FFH & 1506 & 59 & 0.0026 & 0.1609 & 0.2170 & $A_{5}$ \\
\hline FFHOWG & 0.0261 & 0.0796 & -0.0066 & 0.1435 & 0.1742 & $A_{2} \succ$ \\
\hline$F F H$ & 0.7929 & 0.6573 & 0.4 & 0.3184 & 0.1063 & $A_{2} \succ A_{3} \succ A_{4} \succ$ \\
\hline$F F H$ & 4513 & 0.5977 & & 0.7557 & 0.6905 & $A_{5} \succ A_{3} \succ A_{2} \succ A_{1}$ \\
\hline FFHPWA & .7929 & 0.6572 & 0.4948 & 0.3182 & 0.0737 & $A_{2} \succ A_{3} \succ A_{4} \succ A$ \\
\hline$F F H P W G$ & 0.4513 & 0.5978 & 0.6144 & 0.7558 & 0.6909 & $A_{4} \succ A_{5} \succ A_{3} \succ A_{2} \succ A$ \\
\hline
\end{tabular}

The graphical views of these ranking orders are shown in Fig 1. 


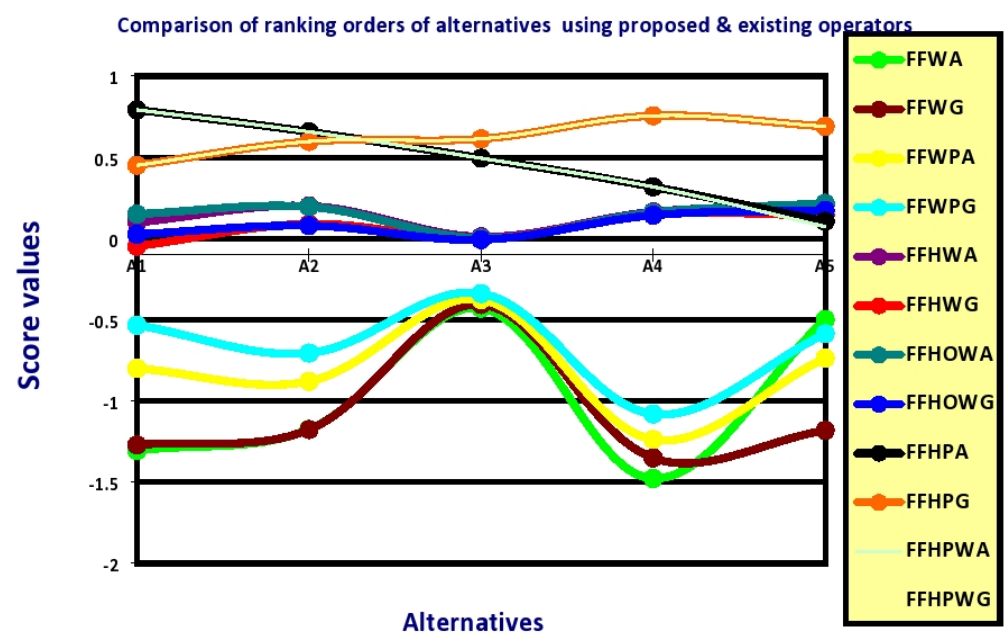

Fig 1. Proposed and existing operators. Line $\mathrm{x}$-axis represents alternatives and y-axis represents score values of alternatives

From Fig 1, we observe that the ranking orders of all the alternatives using several existing and proposed operators are different. We observe that the graphs of existing operators (FFWA (green line), FFWG (brown line), FFWPA (yellow line), and FFWPG (aqua line)) are monotonically increasing \& decreasing between the alternatives $A_{1}$ to $A_{5}$, and we can't observe stability in these operators. On the other hand, the graphs of proposed operators (FFHWA (purple line), FFHWG (red line), FFHOWA (teal line), and FFHWG (black line)) are more stable and their graphs have very rare fluctuations. Therefore, the proposed operators are seems to be more stable.

\subsubsection{Effect of prioritizations of attributes}

In Table 3, the score values and their ranking orders of alternatives in FFHPA, FFHPG, FFHPWA and FFHPWG operators are given. 
Table 3. Ranking order in proposed operators

\begin{tabular}{ccc}
\hline Alternatives & FFHPA & FFHPG \\
\hline$A_{1}$ & 0.7929 & 0.4513 \\
$A_{2}$ & 0.6573 & 0.5977 \\
$A_{3}$ & 0.4949 & 0.6142 \\
$A_{4}$ & 0.3184 & 0.7557 \\
$A_{5}$ & 0.1063 & 0.6905 \\
Ranking $A_{1} \succ A_{2} \succ A_{3} \succ A_{4} \succ A_{5} \quad A_{4} \succ A_{5} \succ A_{3} \succ A_{2} \succ A_{1}$ \\
\hline \multicolumn{2}{c}{ FFHPWA } & FFHPWG \\
\hline Alternatives & 0.7929 & 0.4513 \\
$A_{1}$ & 0.6572 & 0.5978 \\
$A_{2}$ & 0.4948 & 0.6144 \\
$A_{3}$ & 0.3184 & 0.7558 \\
$A_{4}$ & 0.0737 & 0.6909 \\
$A_{5}$ & $A_{1} \succ A_{2} \succ A_{3} \succ A_{4} \succ A_{5}$ & $A_{4} \succ A_{5} \succ A_{3} \succ A_{2} \succ A_{1}$ \\
Ranking &
\end{tabular}

From Table 3, we observe that the ranking order of alternatives in FFHPA and FFHPWA operators have very rare fluctuations. Similarly, the ranking orders in FFHPG and FFHPWG operator have very small changes. It is concluded that the weights of alternatives in prioritized aggregation operators of FF-sets have a very low effect on the order of alternatives based on Hamacher operations.

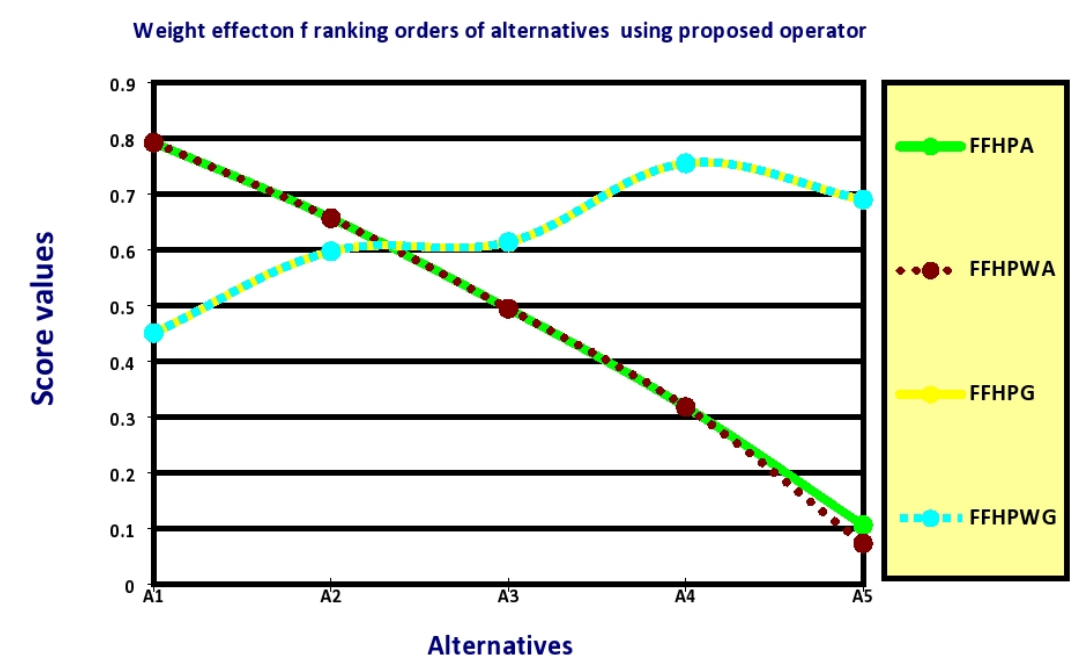

Fig 2. Comparison of proposed operators. $\mathrm{x}$-axis represents alternatives $\mathrm{y}$-axis represents score values

From Fig 2, it is clear that the ranking order of alternatives in (FFHPA) (green line) and 
(FFHPWA) (doted line) are approximately parallel. While the ranking order in (FFHPG) (yellow line) and (FFHPWG) (dotted line) are apparently parallel. This means that the weights in Fermatean fuzzy Hamacher (prioritized) weighted avaeraging and (prioritized) weighted geometric operators have very small effect on the ranking order of alternatives. It is also observed that the ranking order is monotonically increasing in FFHPA and FFHPWA operators while monotonically decreasing in FFHPG and FFHPWG operators.

To compare our proposed operators with the results obtained by applying Pythagorean fuzzy aggregation operators with and without prioritization of weights of attributes, we consider the methods proposed in [19] and [29].

In [19], Pythagorean fuzzy Hamacher weighted averaging and geometric agrregation operators have been applied, if we consider the attribute weight of alternatives as $\Phi=(0.1,0.2,0.3,0.4)^{T}$, then the score values of alternative are given in the following table.

Table 4. Score values of alternatives using PFHWA and PFHWG operator

\begin{tabular}{ccc}
\hline Alternatives & PFHWA & PFHWG \\
$A_{1}$ & 0.2323 & 0.0071 \\
$A_{2}$ & 0.0161 & -0.2471 \\
$A_{3}$ & 0.3704 & 0.1563 \\
$A_{4}$ & 0.2438 & 0.0108 \\
$A_{5}$ & 0.0062 & -0.3125 \\
Ranking & $A_{3} \succ A_{4} \succ A_{1} \succ A_{2} \succ A_{5}$ & $A_{3} \succ A_{4} \succ A_{1} \succ A_{2} \succ A_{5}$
\end{tabular}

On the other hand, if we consider the prioritization of attributes and use PFHPWA and PFHPWG operators, then the score values and their corresponding ranking orders are shown in Table 5 .

Table 5. Score values of alternatives using PFHPWA and PFHPWG operator

\begin{tabular}{ccc}
\hline Alternatives & PFHPWA & PFHPWG \\
$A_{1}$ & 0.6522 & 0.5496 \\
$A_{2}$ & 0.4816 & 0.3618 \\
$A_{3}$ & 0.6228 & 0.4888 \\
$A_{4}$ & 0.5964 & 0.4918 \\
$A_{5}$ & 0.4648 & 0.3568 \\
Ranking & $A_{1} \succ A_{3} \succ A_{4} \succ A_{2} \succ A_{5}$ & $A_{1} \succ A_{4} \succ A_{3} \succ A_{2} \succ A_{5}$ \\
\hline
\end{tabular}

Ranking order of alternatives using PFHPWA and PFHPWG operators and proposed operators with weighted prioritization are shown in Tables 6 \& 7 . 
Table 6. Score values of alternatives using PFHPWA and FFHPWG operator

\begin{tabular}{ccc}
\hline Alternatives & PFHPWA & FFHPWA \\
$A_{1}$ & 0.6522 & 0.7929 \\
$A_{2}$ & 0.4816 & 0.6572 \\
$A_{3}$ & 0.6228 & 0.4948 \\
$A_{4}$ & 0.5964 & 0.3184 \\
$A_{5}$ & 0.4648 & 0.0737 \\
Ranking & $A_{1} \succ A_{3} \succ A_{4} \succ A_{2} \succ A_{5}$ & $A_{1} \succ A_{2} \succ A_{3} \succ A_{4} \succ A_{5}$ \\
\hline
\end{tabular}

Table 7. Score values of alternatives using PFHPWG and FFHPWG operator

\begin{tabular}{ccc}
\hline Alternatives & PFHPWG & FFHPWG \\
$A_{1}$ & 0.5496 & 0.4513 \\
$A_{2}$ & 0.3618 & 0.5978 \\
$A_{3}$ & 0.4888 & 0.6144 \\
$A_{4}$ & 0.4918 & 0.7558 \\
$A_{5}$ & 0.3568 & 0.6909 \\
Ranking & $A_{1} \succ A_{4} \succ A_{3} \succ A_{2} \succ A_{5}$ & $A_{4} \succ A_{5} \succ A_{3} \succ A_{2} \succ A_{1}$ \\
\hline
\end{tabular}

In the following figures, we compare our proposed operators with the existing operators of Pythagorean fuzzy Hamacher averaging and geometric operators with prioritization of attributes.

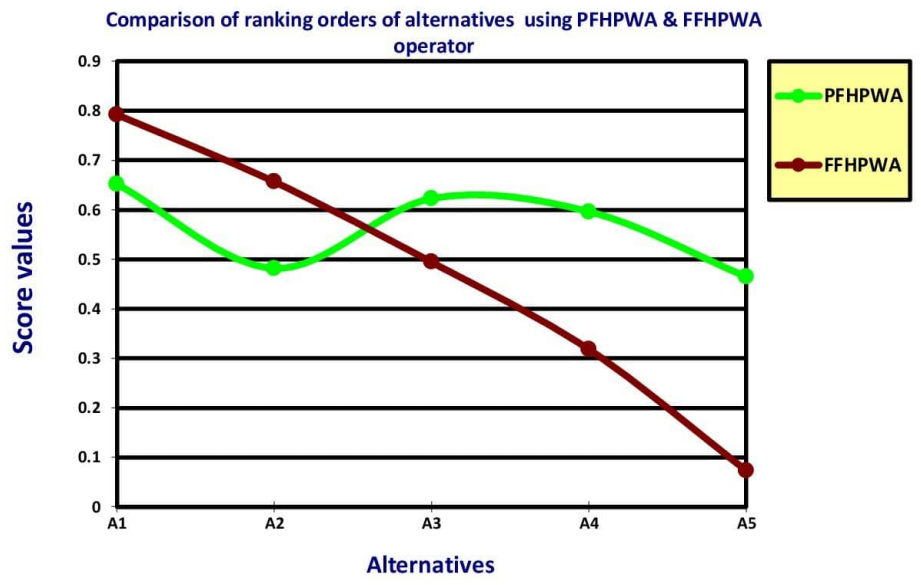

Fig 3. Comparison of PFHPWA and FFHPWA operator. Alternatives are represented by $\mathrm{x}$-axis and score values are represented by $\mathrm{y}$-axis 


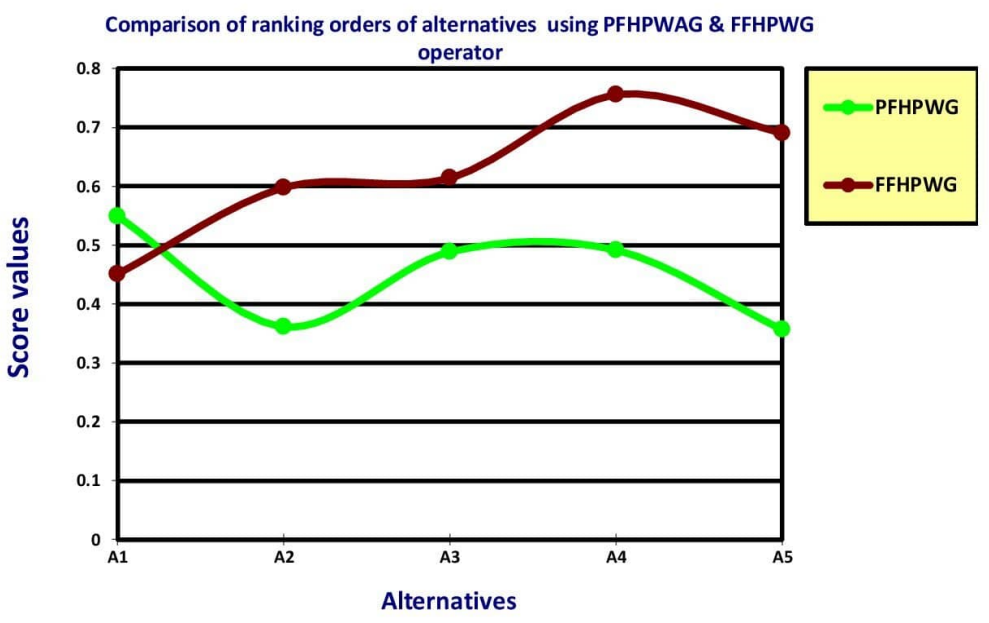

Fig 4. Comparison graphs of PFHPWG and FFHPWG operators. Alternatives are represented by $\mathrm{x}$-axis and score values are represented by $\mathrm{y}$-axis.

From Fig 3, the ranking order of alternatives using PFHPWA operator (green line) smoothly decreasing, while the ranking of alternatives using FFHPWA operator (brown line) are strictly decreasing.

In Fig 4, the ranking order of alternatives in PFHPWG operator (green line) are smoothly decreasing while the ranking order of alternatives in FFHPWG operator (brown line) are smoothly increasing.

\section{Concluding remarks}

In this paper, we have examined a MADM phenomena for Fermatean fuzzy information using Hamacher operations with prioritization of attributes. We presented here arithmetic and geometric operations to initiate some Fermatean fuzzy Hamacher prioritized aggregation operators from the rationale of Hamacher operations as Fermatean fuzzy Hamacher prioritized average (FFHPA) operator, Fermatean fuzzy Hamacher prioritized weighted average (FFHPWA) operator, Fermatean fuzzy Hamacher prioritized geometric (FFHPG) operator, and Fermatean fuzzy Hamacher prioritized weighted geometric (FFHPWG) operator. Several new aspects of these recommended operators are considered. As a fact check, we have applied these operators to look into strategies remedying MADM situations. Eventually, a genuine example for cyclone disasters is considered to develop a strategy and usefulness about the presented method. The new operators are compared with Pythagorean fuzzy Hamacher aggregation operators which gave the reliability of these operators. In future, we will consider decision-making theory, risk theory and other areas under uncertain conditions for the proposed Fermatean fuzzy sets models. 


\section{Author's contribution}

Khan and Khan discussed and formulated the measures. Jan and Afridi wrote the paper together.

\section{Conflict of interest}

The authors declare no conflict of interest.

\section{Ethical approval}

This article does not contain any studies with human participants or animal performed by any of the authors.

\section{References}

[1] K. T. Atanassov, Intuitionistic fuzzy sets. Fuzzy Sets Syst. 20 (1986): 87-96.

[2] K. T. Atanassov, More on intuitionistic fuzzy sets. Fuzzy Sets Syst. 33 (1989): 37-46.

[3] G. Beliakov, A. Pradera, T. Calvo, aggregation Functions: A Guide For Practitioners. Springer, Heidelberg (2007).

[4] H. Garg, A new generalized pythagorean fuzzy information aggregation using Einstein operations and its application to decision making. Int. J. Intell. Syst. 31 (2016): 886-920.

[5] X. Gou, Z. Xu, P. Ren, The properties of continuous Pythagorean fuzzy information. Int. J. Intell. Syst. 31 (2016): 401-424.

[6] H. Hamachar, Uber logische verknunpfungenn unssharfer Aussagen und deren Zugenhorige Bewertungsfunktione Trappl, Klir, Riccardi (Eds), Progress in Cybernatics and Systems Research. 3 (1978): 276-288.

[7] W. Li, Approaches to decision making with interval-valued intuitionistic fuzzy information and their application to enterprise financial performance assessment. J. Intell. Fuzzy Syst. 27(1) (2014): 1-8 .

[8] P.D. Liu, Some Hamacher aggregation operators based on the interval-valued intuitionistic fuzzy numbers and their application to group decision making. IEEE Trans. Fuzzy Syst. 22(1) (2014): 83-97.

[9] R. Parvathi, 2005. Theory of Operators on Intuitionistic Fuzzy Sets of Second Type and their Applications to Image Processing (Ph.D. dissertation). Dept. Math. Alagappa Univ., Karaikudi, India.

[10] R. Parvathi, P. Vassilev, K.T. Atanassov, A note on the bijective correspondence between intuitionistic fuzzy sets and intuitionistic fuzzy sets of pth type. In: New Developments in Fuzzy Sets, Intuitionistic Fuzzy Sets, Generalized Nets and Related Topics. Volume I: Foundations. SRI PAS IBS PAN, Warsaw, 2012, pp. 143-147. 
[11] X. Peng, Y. Yang, Some results for pythagorean fuzzy sets. Int. J. Intell. Syst. 30, (2015): $1133-1160$.

[12] M.Z. Reformat, R.R. Yager, Suggesting recommendations using pythagorean fuzzy sets illustrated using netflix movie data. In: Information Processing and Management of Uncertainty in Knowledge-Based Systems. Springer, 2014. pp. 546-556.

[13] P. Ren, Z. Xu, X. Gou, Pythagorean fuzzy TODIM approach to multi-criteria decision making. Appl. Soft Comput. 42 (2016): 246-259.

[14] T. Senapati, R.R. Yager, Fermatean fuzzy sets. J. Ambient Intell. Hum. Comput. minor revision submitted for publication 2019a.

[15] T. Senapati, R.R. Yager, Some new operations over Fermatean fuzzy numbers and application of Fermatean fuzzy WPM in multi criteria decision making. Informatica 30 (2) (2019b): 391-412.

[16] T. Senapati, R. R. Yager, Fermatean fuzzy weighted averaging/geometric operators and its application in multi-criteria decision-making methods, Engineering Applications of Artificial Intelligence 85 (2019): 112-121.

[17] C.Q. Tan, W.T. Yi, X.H. Chen, Hesitant fuzzy Hamacher aggregation operators for multicriteria decision making. Appl. Soft Comput. 26 (2015): 325-349.

[18] G. Wei, F. E. Alsaadi T. Hayat, A. Alsaedi, Bipolar Fuzzy Hamacher aggregation Operators in multi Attribute Decision Making, Int. J. Fuzzy Syst. DOI 10.1007/s40815-017$0338-6$.

[19] S. J. Wu, G. W. Wei, Pythagorean fuzzy Hamacher aggregation operators and their application to multi attribute decision making, International Journal of Knowledge-based and Intelligent Engineering Systems 21 (2017): 189-201.

[20] S. Xiao, Induced interval-valued intuitionistic fuzzy Hamacher ordered weighted geometric operator and their application to multi attribute decision making. J. Intell. Fuzzy Syst. 27(1) (2014): 527-534.

[21] Z. S. Xu, R. R. Yager, Some geometric aggregation operators based on intuitionistic fuzzy sets. Int. J. Gen Syst. 35 (2006): 417-433.

[22] Z. S. Xu, Intuitionistic fuzzy aggregation operators. IEEE Trans. Fuzzy Syst. 15(6) (2007): 1179-1187.

[23] Z. S. Xu, R. R. Yager, Dynamic intuitionistic fuzzy multi-attribute decision making. Int. J. Approx. Reason. 48(1) (2008): 246-262.

[24] Z.S. Xu, M.M. Xia, Induced generalized intuitionistic fuzzy operators. Knowl. Based Syst. 24(2) (2011): 197-209 . 
[25] Z.S. Xu, Q. Chen, A multi-criteria decision making procedure based on intuitionistic fuzzy bonferroni means. J. Syst. Sci. Syst. Eng. 20(2) (2011): 217-228.

[26] Z.S. Xu, Approaches to multi attribute group decision making based on intuitionistic fuzzy power aggregation operators. Knowl. Based Syst. 24(6) (2011): 749-760.

[27] R.R. Yager, A.M. Abbasov, Pythagorean membership grades, complex numbers, and decision making. Int. J. Intell. Syst. 28 (2013): 436-452.

[28] L. A. Zadeh, Fuzzy sets. Inf. Control 8 (1965): 338-356.

[29] S. Zeng, Z. Mu, T. Balezentis, A novel aggregation method for Pythagorean fuzzy multi attribute group decision making. Int. J. Intell. Syst. 33 (3) (2018): 573-585.

[30] X. Zhang, A novel approach based on similarity measure for pythagorean fuzzy multi criteria group decision making. Int. J. Intell. Syst. 31 2016, 593-611.

[31] X. Zhang, Z. Xu, Extension of TOPSIS to multi criteria decision making with Pythagorean fuzzy sets. Int. J. Intell. Syst. 29 (2014): 1061-1078.

[32] L.Y. Zhou, X.F. Zhao, G.W. Wei, Hesitant fuzzy hamacher aggregation operators and their application to multi attribute decision making. J. Intell. Fuzzy Syst. 26(6) (2014): 2689-2699.

[33] T. -Y. Chen, Nonlinear assignment-based methods for interval-valued intuitionistic fuzzy multi-criteria decision-making analysis with incomplete preference information, Int'l J Inf Tech \& decision Making 11 (2012), 821-855.

[34] T. - Y. Chen, The extended linear assignment methods for multiple criteria decisionmaking based on interval-valued intuitionistic fuzzy sets, Appl Math Model 38 (2014), 2101-2117.

[35] T. -Y. Chen, Interval-valued intuitionistic fuzzy QUALIFLEX method with a likelihoodbased comparison approach for multiple criteria decision analysis, Inf Sci 261 (2014), 149-169.

[36] T.-Y. Chen, An interval-valued Pythagorean fuzzy compromise approach with correlation-based closeness indices for multiple-criteria decision analysis of bridge construction methods, Complexity 2018 (2018).

[37] T.-Y. Chen, A novel PROMETHEE-based outranking approach for multiple criteria decision analysis with Pythagorean fuzzy information, Ieee Access 6 (2018), 54495-54506.

[38] T.Y. Chen, An interval-valued Pythagorean fuzzy outranking method with a closenessbased assignment model for multiple criteria decision making, International Journal of Intelligent Systems 33 (2018), 126-168. 
[39] J.-C. Wang and T.-Y. Chen, Likelihood-based assignment methods for multiple criteria decision analysis based on interval-valued intuitionistic fuzzy sets, Fuzzy Optimization and Decision Making 14 (2015), 425-457.

[40] S. -J. Wu and G.-W. Wei, Pythagorean fuzzy Hamacher aggregation operators and their application to multiple attribute decision making, Inter J Knowledge-based Intell Eng Systs 21 (2017) 189-201.

[41] H. Garg, G. Shahzadi, and M. Akram, Decision-Making Analysis Based on Fermatean Fuzzy Yager Aggregation Operators with Application in COVID-19 Testing Facility, Mathematical Problems in Engineering Volume 2020, Article ID 7279027, 16 pages

[42] S. B. Aydemir and S. Y. Gunduz, Fermatean fuzzy TOPSIS method with dombi aggregation operators and its application in multi-criteria decision making, Journal of Intelligent \& Fuzzy Systems xx (2020) x-xx, DOI:10.3233/JIFS-191763.

[43] A. Hadi, W. Khan and A. Khan, A novel approach to MADM problems using Fermatean fuzzy Hamacher aggregation operators, Int J Intell Syst DOI: 10.1002/int.22324. 